\title{
Iodine, a Mild Reagent for the Aromatization of Terpenoids
}

\author{
Victoriano Domingo, ${ }^{\mathrm{a}}$ Consuelo Prieto, ${ }^{\mathrm{a}}$ Lucia Silva, ${ }^{\mathrm{b}}$ Jesús M. L. Rodilla, ${ }^{\mathrm{b}}$ José
} Francisco Quílez del Moral, ${ }^{\mathrm{a}}$ Alejandro F. Barrero*a

${ }^{a}$ Department of Organic Chemistry, Institute of Biotechnology, Faculty of Sciences, University of Granada, Avda. Fuente Nueva, s/n,18071 Granada, Spain

${ }^{\mathrm{b}}$ FibEnTech-Materiais Fibrosos e Tecnologias Ambientais, Department of Chemistry, University of Beira Interior, Rua Marquês d'Ávila e Bolama, 6200-Covilhã, Portugal. afbarre@ugr.es,jfquilez@ugr.es

\section{Supporting Information}

\section{Table of contents}




\section{Experimental}

Aromatization promoted by iodine: Standard procedure. To a solution of the corresponding allylic/homoallylic alcohol or diene ( $1 \mathrm{mmol}, 1$ equiv) in toluene $(0.1$ $0.25 \mathrm{M})$ at reflux, iodine was added $(0.2-3.0 \mathrm{mmol})$. The solution was stirred at reflux and monitored by thin-layer chromatography analysis. If necessary, a second addition of iodine was added. Upon consumption of the starting material, the reaction was diluted in hexanes $(100 \mathrm{~mL})$ and washed with a saturated solution of sodium thiosulfate $(1 \mathrm{x} 100$ $\mathrm{mL}), \mathrm{NaHCO}_{3}(1 \times 100 \mathrm{~mL})$ and brine $(1 \mathrm{x} 100 \mathrm{~mL})$. The layers were separated, the aqueous layer was extracted with hexanes $(2 \times 50 \mathrm{~mL})$, and the process was repeated. The organic extracts were combined and dried over sodium sulfate and evaporated in vacuo. Purification was performed by silica gel chromatography to yield chromatographically and spectroscopically pure products.

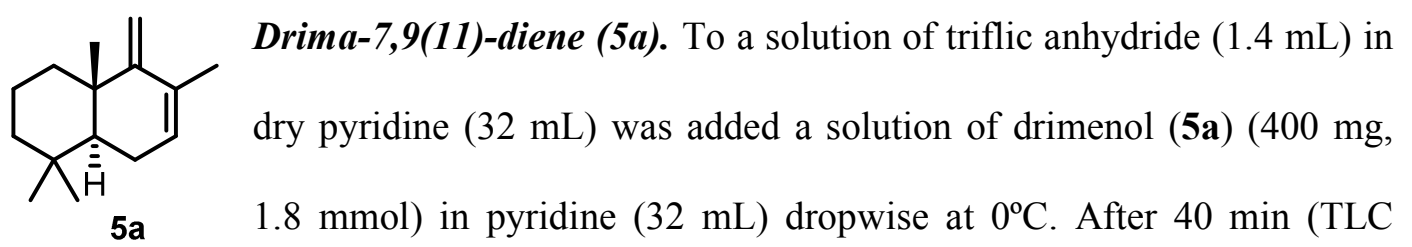
analysis), the reaction mixture was poured over a mixture of ice-water, extracted with $t$ $\mathrm{BuOMe}(200 \mathrm{~mL})$ and washed with a saturated solution of $\mathrm{NaHCO}_{3}(200 \mathrm{~mL})$ and brine $(200 \mathrm{~mL})$ and then dried $\left(\mathrm{Na}_{2} \mathrm{SO}_{4}\right)$ and concentrated under vacuo. The residue was purified by flash chromatography (silica gel, hexanes) to give $\mathbf{5 a}($ exo) and $\mathbf{5 b}$ (endo) in $4: 1$ ratio $(43 \%)$. 
The spectroscopic data for this compound were identical to those reported in the literature: H. Akita, M. Nozawa, A. Mitsuda, H. Ohsawa, Tetrahedron: Asymmetry, 2000, 11, 1375-1388.

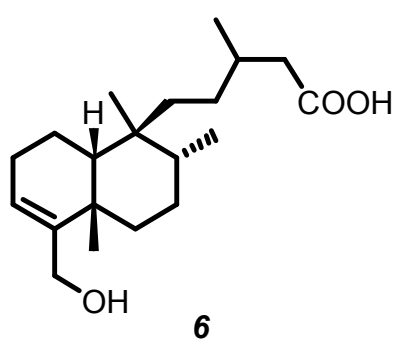

(+)-18-Hydroxy-cis-clerodan-3-ene-15-oic acid (6): E. Kalpoutzakis, N. Aligiannis, Nektarios, A. Skaltsounis, S. Mitakou, J. Nat. Prod. 2003, 66, 316-319.

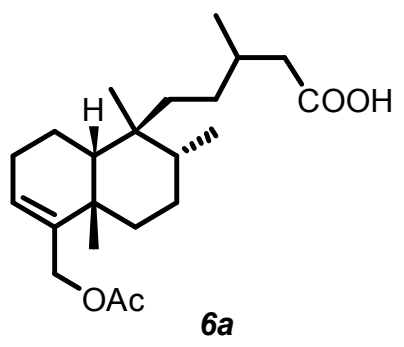

(+)-19-acetoxy-cis-clerodan-3-ene-15-oic acid (6a): A. Kolocouris, T. Mavromoustakos, C. Demetzos, A. Terzis, S. G. Grdadolnik, Bio. Med. Chem. Lett. 2001, 11, 837-840.

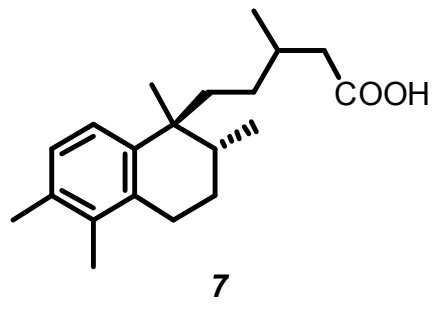

Compound 7: The Standard Procedure was followed with a reaction time of 5 hours. Colorless oil, $[\alpha]^{20}{ }_{\mathrm{D}}=+4.2(\mathrm{c}$ 0.8, $\mathrm{CH}_{2} \mathrm{Cl}_{2}$ ), IR (film): 3426, 2959, 2926, 2871, 1706, 1458, 1299, $813 \mathrm{~cm}^{-1} ;{ }^{1} \mathrm{H}$ NMR (500 MHz, $\left.\mathrm{CDCl}_{3}\right) \delta 7.04$ $(\mathrm{d}, J=8.1 \mathrm{~Hz}, 1 \mathrm{H}), 6.97(\mathrm{~d}, J=8.1 \mathrm{~Hz}, 1 \mathrm{H}), 2.71(\mathrm{dt}, J=17.0,4.7 \mathrm{~Hz}, 1 \mathrm{H}), 2.57(\mathrm{ddd}, J=$ 17.0, 10.0, 6.6 Hz, 1H), 2.33 (dd, J=15.0, $5.4 \mathrm{~Hz}, 1 \mathrm{H}), 2.25$ (s, 3H), $2.12(\mathrm{~s}, 3 \mathrm{H}), 2.05$ $(\mathrm{dd}, \mathrm{J}=15.0,8.7 \mathrm{~Hz}, 1 \mathrm{H}), 1.88-1.57(\mathrm{~m}, 7 \mathrm{H}), 1.12(\mathrm{~s}, 3 \mathrm{H}), 1.08(\mathrm{~m}, 1 \mathrm{H}), 0.93(\mathrm{~d}, J=6.7$ $\mathrm{Hz}, 6 \mathrm{H}) ;{ }^{13} \mathrm{C} \mathrm{NMR}\left(100 \mathrm{MHz}, \mathrm{CDCl}_{3}\right) \delta 177.5,142.3,135.3,134.5,133.2,127.6$, 
124.0, 41.1, 40.3, 37.6, 32.9, 31.0, 31.0, 27.2, 27.0, 26.1, 20.6, 19.9, 16.1, 15.3; HRMS (ESI-TOF) $\mathrm{m} / \mathrm{z}$ calcd for $\mathrm{C}_{20} \mathrm{H}_{31} \mathrm{O}_{2} 303.2319[\mathrm{M}+\mathrm{H}]^{+}$, found 303.2324

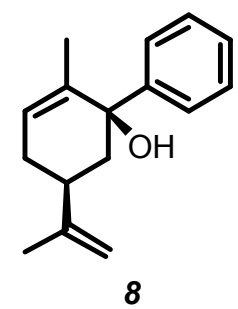

2-Phenylcarveol (8): A $30 \mathrm{ml}$ flask was placed in a water bath (room temperature) charged with magnesium turnings (363 mg, $15 \mathrm{mmol})$ fitted with a rubber septum and purged with Ar. Dry THF (19 mL) was added via syringe followed by a solution of DIBAL-H in THF $(0.1 \mathrm{ml}$ $1 \mathrm{M}$ in THF, $0.001 \mathrm{mmol})$. The mixture was stirred at $\mathrm{rt}$ for $5 \mathrm{~min}$. Then, $(1.05 \mathrm{ml}, 1.05$ mmol) bromobenzene was added dropwise and the reaction stirred for $3 \mathrm{~h}$ at $\mathrm{rt}$. To a solution of $(S)$ - carvone $(100 \mathrm{mg}, 0.665 \mathrm{mmol})$ in $3 \mathrm{~mL}$ of dry $\mathrm{THF}$ at $0^{\circ} \mathrm{C}$ was added the benzylmagnesium bromide prepared previously. After stirring for $3 \mathrm{~h}$ (TLC monitoring), the mixture was diluted with $t$ - $\mathrm{BuOMe}$ and $\mathrm{NH}_{4} \mathrm{Cl}$ and extracted with $t$ BuOMe. The combined organic layer was washed with brine, dried over anhydrous $\mathrm{Na}_{2} \mathrm{SO}_{4}$ and concentrated under reduced pressure. The resulting crude was purified by chromatography (hexane/t-BuOMe, 3:1) on silica gel to afford $110 \mathrm{mg}$ (72\%) of the corresponding aryl derivative 8. Colorless oil, ${ }^{1} \mathrm{H}$ NMR $\left(400 \mathrm{MHz}, \mathrm{CDCl}_{3}\right) \delta 7.47(\mathrm{~d}, J=$ $7.7 \mathrm{~Hz}, 2 \mathrm{H}), 7.35$ (t, $J=7.7 \mathrm{~Hz}, 2 \mathrm{H}), 7.26$ (t, $J=7.7 \mathrm{~Hz}, 1 \mathrm{H}), 5.80$ (brs, 1H), 4.65 (brs, $1 \mathrm{H}), 4.62(\mathrm{~s}, 1 \mathrm{H}), 2.29-1.90(\mathrm{~m}, 5 \mathrm{H}), 1.66(\mathrm{~s}, 3 \mathrm{H}), 1.63(\mathrm{~s}, 3 \mathrm{H}) ;{ }^{13} \mathrm{C}$ NMR (100 MHz, $\left.\mathrm{CDCl}_{3}\right) \delta 148.7,145.7,135.9,128.0(2 \mathrm{C}), 127.3,126.3,126.0(2 \mathrm{C}), 109.0,77.2,45.3$, $37.9,31.2,20.7,17.9$. 


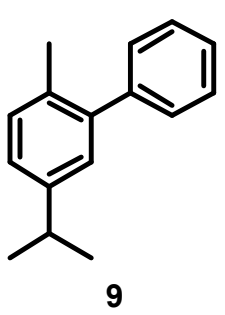

Compound 9: The Standard Procedure was followed with a reaction time of 2 hours. The spectroscopic data for this compound were identical to those reported in the literature: L. Majidi, R. Fihi, Phys. Chem. News, 2004, 15, 83-86. Yellowish oil, ${ }^{1} \mathrm{H}$ NMR (400 MHz, $\left.\mathrm{CDCl}_{3}\right) \delta 7.48-7.12(\mathrm{~m}, 8 \mathrm{H}), 2.95(\mathrm{spt}, J=7.0 \mathrm{~Hz}, 1 \mathrm{H}), 2.28(\mathrm{~s}, 3 \mathrm{H}), 1.30$ (d, $J=7.0 \mathrm{~Hz}$, $6 \mathrm{H}) ;{ }^{13} \mathrm{C} \mathrm{NMR}\left(100 \mathrm{MHz}, \mathrm{CDCl}_{3}\right) \delta 146.5,142.4,141.9,132.7,130.4,129.4,128.2$, $128.1,126.8,125.4,77.5,77.2,76.8,33.9,24.2,20.1$.

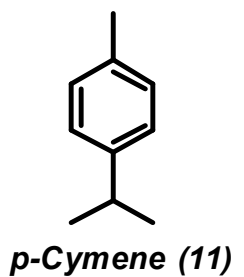

p-Cymene (11): The Standard Procedure was followed with a reaction time of $10 \mathrm{~min}$. Commercially available.

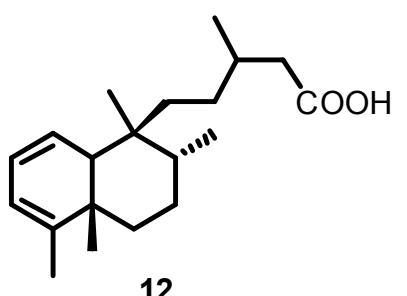

Compound 12: J. De Pascual Teresa; J. G. Urones; J. A. Herrero, An. Quim. 1978, 74, 476-480.

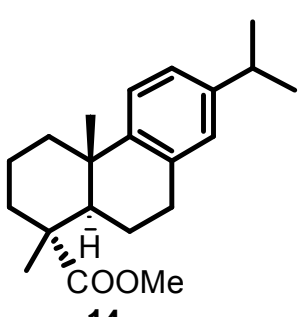

14

Methyl dehydroabietate (14): The Standard Procedure was followed with a reaction time of 6 hours . The spectroscopic data for this compound were identical to those reported in the literature: Alex R. Jurgens; James D. McChesney, J. Magn Reson Chem. 1990, 28, 181-184. 


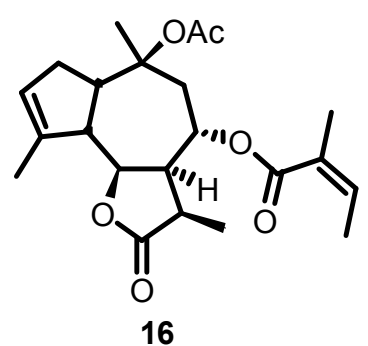

16

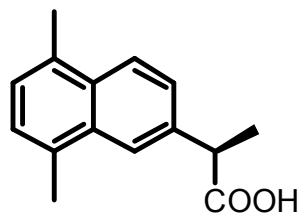

(-)-Naproxen analogue (17): The Standard Procedure was followed with a reaction time of 3.5 hours . Colorless oil, $[\alpha]^{20}{ }_{D}=-$ 54.1 (c 1.2, EtOH), IR (film): 3020, 29323, 2858, 1705, 1458, 1229, 1050, $832 \mathrm{~cm}^{-1} ;{ }^{1} \mathrm{H}$ NMR (500 MHz, $\left.\mathrm{CDCl}_{3}\right) \delta 7.93(\mathrm{~d}, J=8.6 \mathrm{~Hz}, 1 \mathrm{H}), 7.86(\mathrm{~s}$, 1H), 7.37 (dd, $J=8.6,1.8 \mathrm{~Hz}, 1 \mathrm{H}), 7.35(\mathrm{~d}, J=7.5 \mathrm{~Hz}, 1 \mathrm{H}), 7.23(\mathrm{~d}, J=7.5 \mathrm{~Hz}, 1 \mathrm{H})$, $4.51(\mathrm{q}, J=7.0 \mathrm{~Hz}, 1 \mathrm{H}), 2.65(\mathrm{~s}, 3 \mathrm{H}), 2.54(\mathrm{~s}, 3 \mathrm{H}), 1.65(\mathrm{~d}, J=7.0 \mathrm{~Hz}, 3 \mathrm{H}){ }^{13} \mathrm{C} \mathrm{NMR}$ $\left(100 \mathrm{MHz}, \mathrm{CDCl}_{3}\right) \delta 179.3,135.8,134.1,133.7,131.6,131.4,127.9,125.7,125.1$, 124.4, 122.8, 40.8, 22.2, 19.7, 18.1; HRMS (ESI-TOF) $\mathrm{m} / \mathrm{z}$ calc. for $\mathrm{C}_{15} \mathrm{H}_{15} \mathrm{O}_{2} 227.1078$ $[\mathrm{M}-\mathrm{H}]^{+}$, found 227.1072 .

Aromatization promoted by iodine/DDQ: Standard procedure. To a solution of the corresponding allylic alcohol/diene $(1 \mathrm{mmol}$, lequiv) in toluene $(0.1-\mathrm{M})$ at reflux was added iodine $(0.5 \mathrm{mmol})$ and DDQ $(0.5 \mathrm{mmol})$. The solution was stirred at reflux and monitored by thin-layer chromatography analysis. Upon consumption of the starting material the reaction was diluted in hexanes $(100 \mathrm{~mL})$ and filtered through a Buchner funnel with a sintered disk, washed with a saturated solution of sodium thiosulfate (1x $100 \mathrm{~mL}), \mathrm{NaHCO}_{3}(1 \times 100 \mathrm{~mL})$ and brine $(1 \times 100 \mathrm{~mL})$. The layers were separated and the aqueous layer was extracted with hexanes $(2 \times 50 \mathrm{~mL})$. The organic extracts were 
combined and dried over sodium sulfate and evaporated in vacuo. Purification was performed by silica gel chromatography to yield chromatographically and spectroscopically pure product.

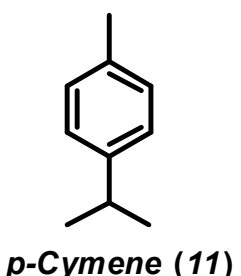

p-Cymene (11): Starting from terpinen-4-ol (10), the Standard Procedure was followed with a reaction time of 1 hour . Starting from limonene (19), the Standard Procedure was followed with a reaction p-Cymene (11) time of $45 \mathrm{~min}$. Commercially available.

Aromatization promoted by iodine/DMSO: Standard procedure. To a solution of the corresponding starting material $(1 \mathrm{mmol}, 1$ equiv) in toluene $(0.1 \mathrm{M})$ at reflux was added iodine $(0.2-1.0 \mathrm{mmol})$ and DMSO $(500 \% \mathrm{~mol})$. The solution was stirred at reflux and monitored by thin-layer chromatography analysis. Upon consumption of the starting material the reaction was diluted in $t$-BuOMe $(100 \mathrm{~mL})$, washed with a saturated solution of sodium thiosulfate $(1 \times 100 \mathrm{~mL})$, and brine $(1 \times 100 \mathrm{~mL})$. The organic layer was dried over sodium sulfate and evaporated in vacuo. Purification was performed by silica gel chromatography to yield chromatographically and spectroscopically pure product.

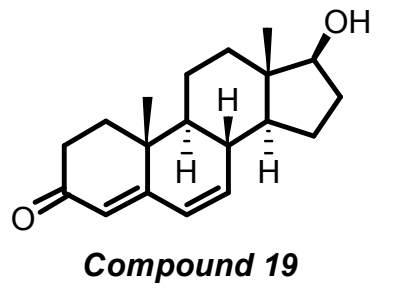

Compound 19: The standard procedure was followed with a reaction time of 3 hours. ${ }^{1} \mathrm{H} \mathrm{NMR}\left(300 \mathrm{MHz}, \mathrm{CDCl}_{3}\right) \delta 6.09$ (bs, 2H), $5.66(\mathrm{bs}, 1 \mathrm{H}), 3.67(\mathrm{t}, J=8.0 \mathrm{~Hz}, 1 \mathrm{H}), 2.56(\mathrm{ddd}, J=$ 
18.0, 14.2, $5.3 \mathrm{~Hz}, 1 \mathrm{H}), 2.41$ (ddd, $J=18.0,5.3,1.4 \mathrm{~Hz}, 1 \mathrm{H}), 2.28-1.05$ (m, 13H), 1.11 (s, 3H), $0.83(\mathrm{~s}, 3 \mathrm{H}) ;{ }^{13} \mathrm{C} \mathrm{NMR}\left(75 \mathrm{MHz}, \mathrm{CDCl}_{3}\right) \delta 199.9,164.2,140.8,128.2,123.8$, $81.4,51.0,48.5,44.1,37.9,36.6,36.3,34.1,30.5,23.2,20.5,16.5,11.3$. The spectroscopic data for this compound were identical to those reported in the literature: E. Kim, E. Ma, Steroids. 2007, 72, 360-367.

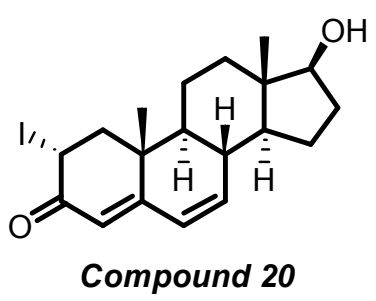

Compound 20: The standard procedure was modified by adding 1 equiv of iodine, with a reaction time of 4 hours to give $15 \%$ of starting material, $61 \%$ of the iodo derivative $\mathbf{2 0}$ and $5 \%$ of the trienone 21. Compound 20: Colorless oil, $[\alpha]^{20}$ $=+83.5$ (c 1.0, $\left.\mathrm{CH}_{2} \mathrm{Cl}_{2}\right)$, IR (film): 3422, 2938, 2869, 1663, 1613, 1582, 1455, 1381, 1225, 1055, $887 \mathrm{~cm}^{-1} ;{ }^{1} \mathrm{H}$ NMR $\left(600 \mathrm{MHz}, \mathrm{CDCl}_{3}\right) \delta 6.16(\mathrm{dd}, J=9.7,2.0 \mathrm{~Hz}, 1 \mathrm{H})$, $6.11(\mathrm{dd}, J=9.8,2.6 \mathrm{~Hz}, 1 \mathrm{H}), 5.74(\mathrm{~s}, 1 \mathrm{H}), 5.29(\mathrm{dd}, \mathrm{J}=14.0,5.4 \mathrm{~Hz}, 1 \mathrm{H}), 3.70(\mathrm{t}, J=$ $8.4 \mathrm{~Hz}, 1 \mathrm{H}), 2.70(\mathrm{dd}, J=13.0,5.4 \mathrm{~Hz}, 1 \mathrm{H}), 2.39(\mathrm{t}, J=13.6 \mathrm{~Hz}, 1 \mathrm{H}), 2.21(\mathrm{t}, J=10.8$ Hz, 1H), 2.17-2.09 (m, 1H), $1.90(\mathrm{dt}, J=12.9,3.4 \mathrm{~Hz}, 1 \mathrm{H}), 1.83-1.76(\mathrm{~m}, 1 \mathrm{H}), 1.62$ $(\mathrm{dq}, J=13.4,3.8 \mathrm{~Hz}, 1 \mathrm{H}), 1.54-1.39(\mathrm{~m}, 3 \mathrm{H}), 1.28-1.10(\mathrm{~m}, 3 \mathrm{H}), 1.17(\mathrm{~s}, 3 \mathrm{H}), 0.84(\mathrm{~s}$, $3 \mathrm{H}) ;{ }^{13} \mathrm{C} \mathrm{NMR}\left(75 \mathrm{MHz}, \mathrm{CDCl}_{3}\right) \delta 192.4,164.1,141.7,127.7,120.8,81.4,50.7,48.9$, 48.3, 44.1, 40.3, 37.8, 36.4, 30.6, 29.7, 23.2, 20.5, 16.8, 11.3; HRMS (ESI-TOF) m/z calc. for $\mathrm{C}_{19} \mathrm{H}_{26} \mathrm{O}_{2} \mathrm{I} 413.0977[\mathrm{M}+\mathrm{H}]^{+}$, found 413.0963 .

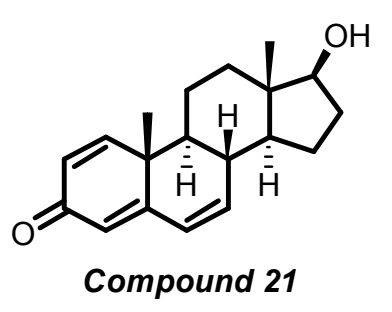

Compound 21: Treatment of the iodo derivative 20 with 3 mmol of DBU at refluxing tolune for $3 \mathrm{~min}$ afforded compound 21: ${ }^{1} \mathrm{H}$ NMR $\left(300 \mathrm{MHz}, \mathrm{CDCl}_{3}\right) \delta 7.09(\mathrm{~d}, J=10.1$ 
$\mathrm{Hz}, 1 \mathrm{H}), 6.27(\mathrm{dd}, J=10.1,1.9 \mathrm{~Hz}, 1 \mathrm{H}), 6.26(\mathrm{dd}, J=9.8,2.9 \mathrm{~Hz}, 1 \mathrm{H}), 6.06-6.01(\mathrm{~m}$, 2H), $3.70(\mathrm{t}, J=7.9 \mathrm{~Hz}, 1 \mathrm{H}), 2.34(\mathrm{bt}, J=10.4 . \mathrm{Hz}, 1 \mathrm{H}), 2.21-1.10(\mathrm{~m}, 10 \mathrm{H}), 1.23(\mathrm{~s}$, 3H), $0.89(\mathrm{~s}, 3 \mathrm{H}) ;{ }^{13} \mathrm{C} \mathrm{NMR}\left(75 \mathrm{MHz}, \mathrm{CDCl}_{3}\right) \delta 186.6,164.9,153.3,138.0,128.3$, $128.0,124.2,81.4,51.0,48.7,48.7,43.7,41.5,38.4,36.5,30.5,23.2,21.7,21.0,11.4$. The spectroscopic data for this compound were identical to those reported in the literature: E. Kim, E. Ma, Steroids. 2007, 72, 360-367. 
proton

12-1083_P-965-1

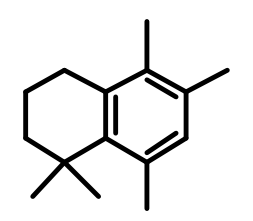

2

${ }^{1} \mathrm{H}$ NMR $\left(\mathrm{CDCl}_{3}\right), 500 \mathrm{MHz}$

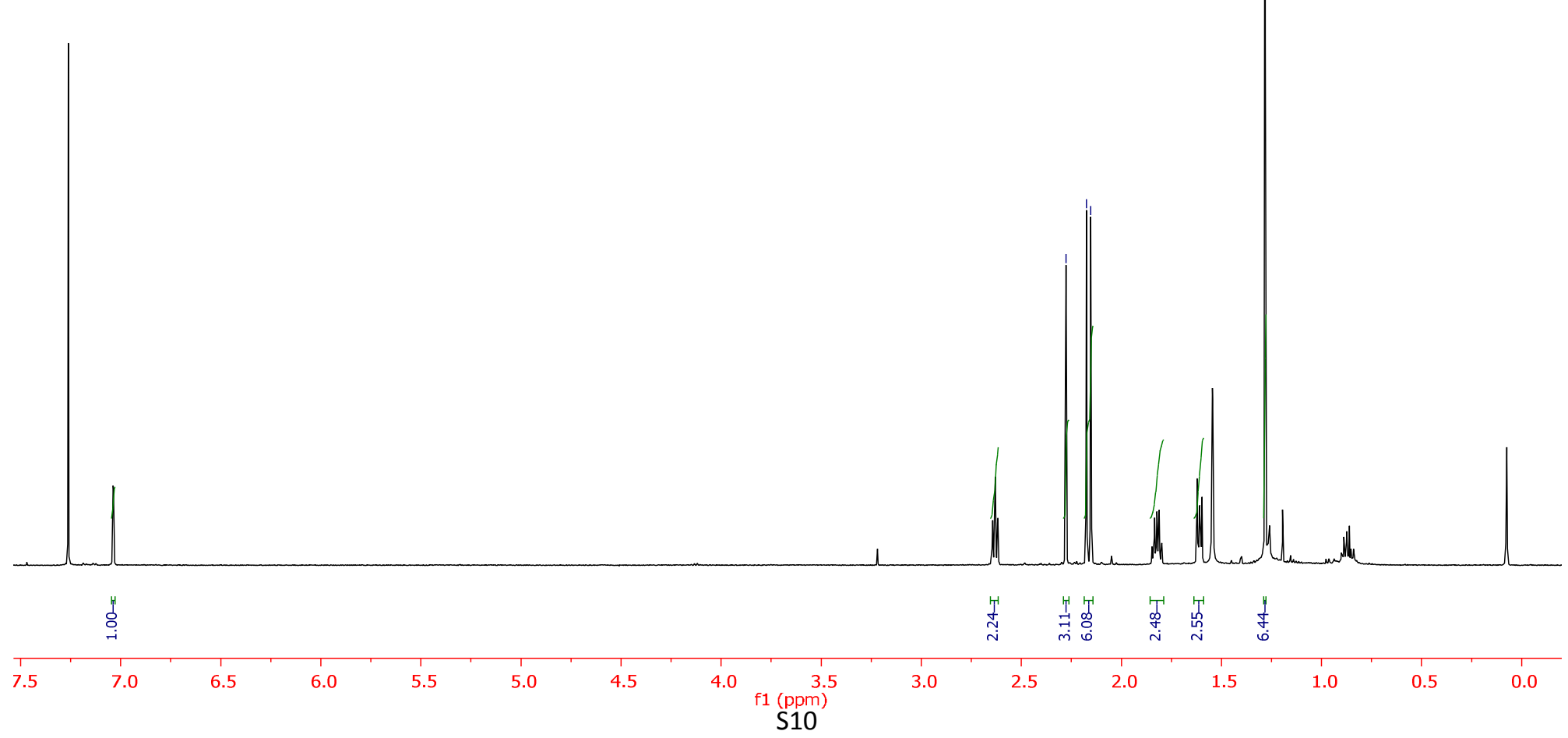




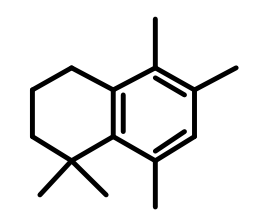

2

${ }^{13} \mathrm{C} \mathrm{NMR}\left(\mathrm{CDCl}_{3}\right), 125 \mathrm{MHz}$
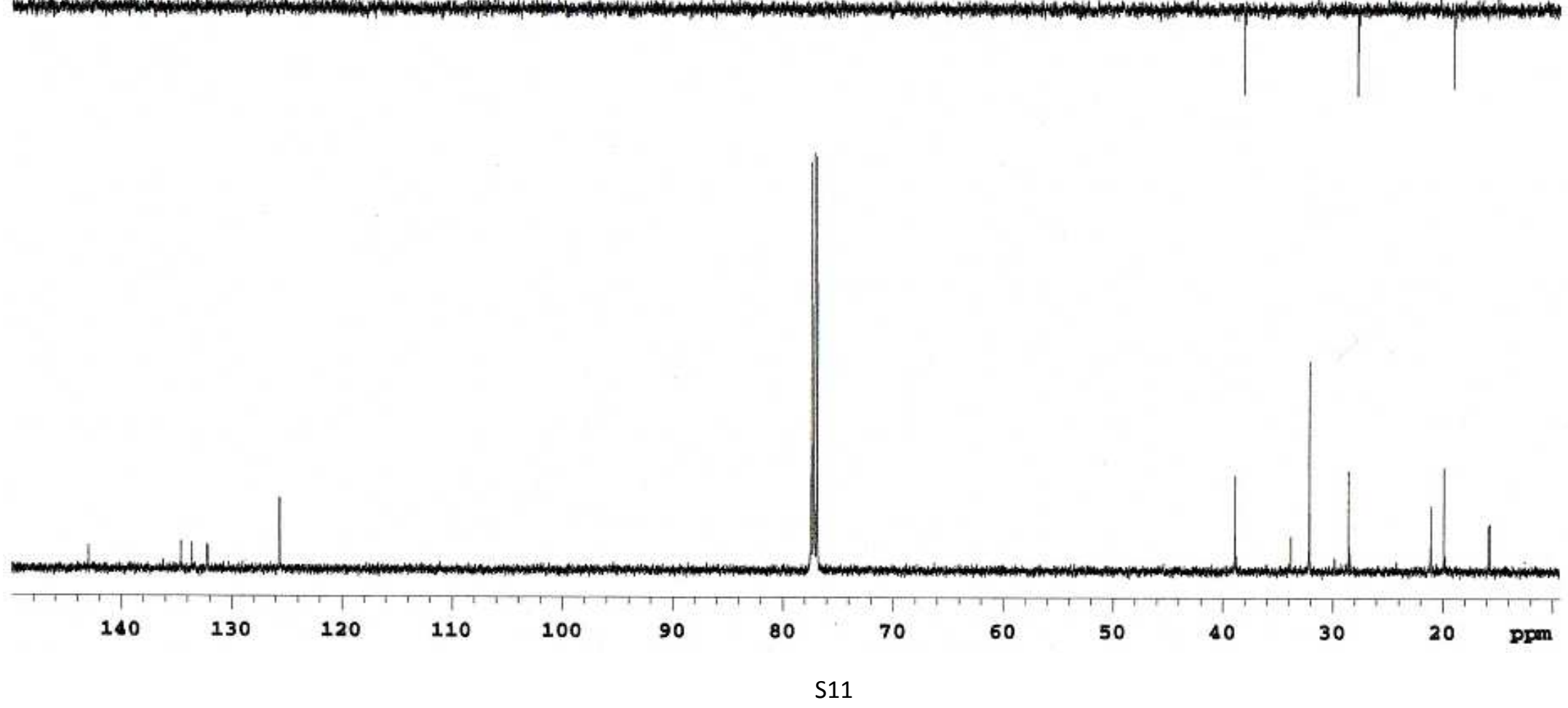


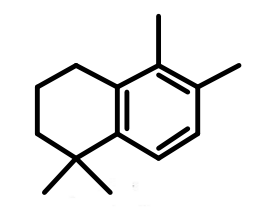

${ }^{1} \mathrm{H} \mathrm{NMR}\left(\mathrm{CDCl}_{3}\right), 500 \mathrm{MHz}$

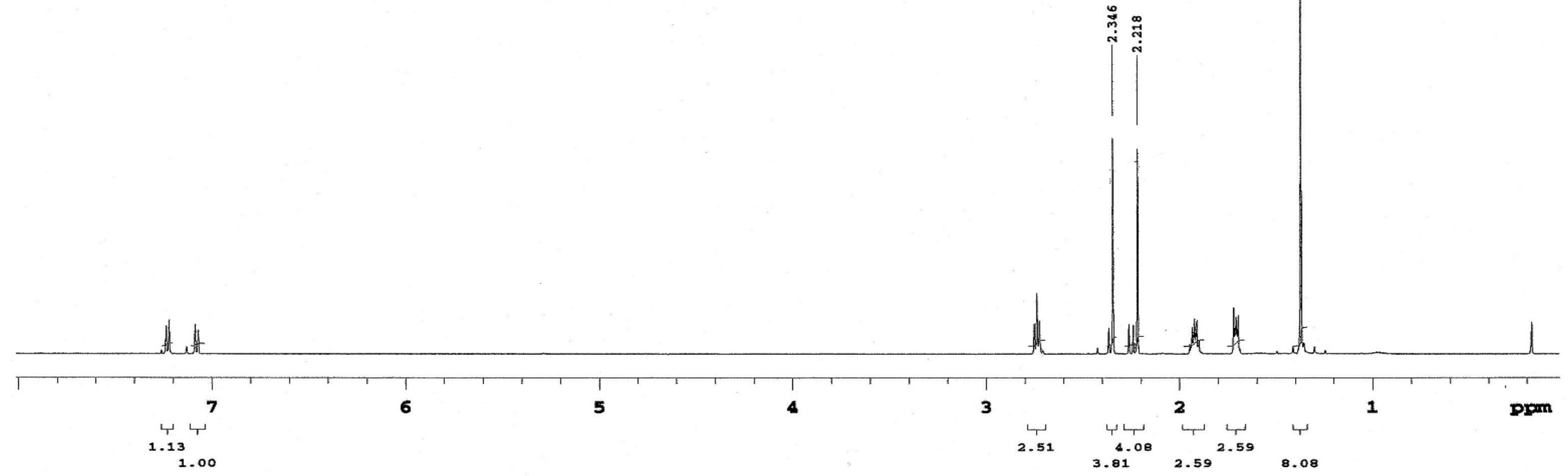




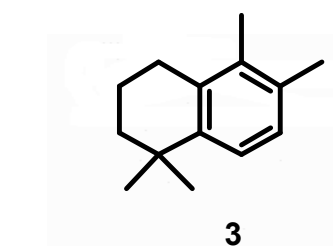

${ }^{13} \mathrm{C} \mathrm{NMR}\left(\mathrm{CDCl}_{3}\right), 125 \mathrm{MHz}$
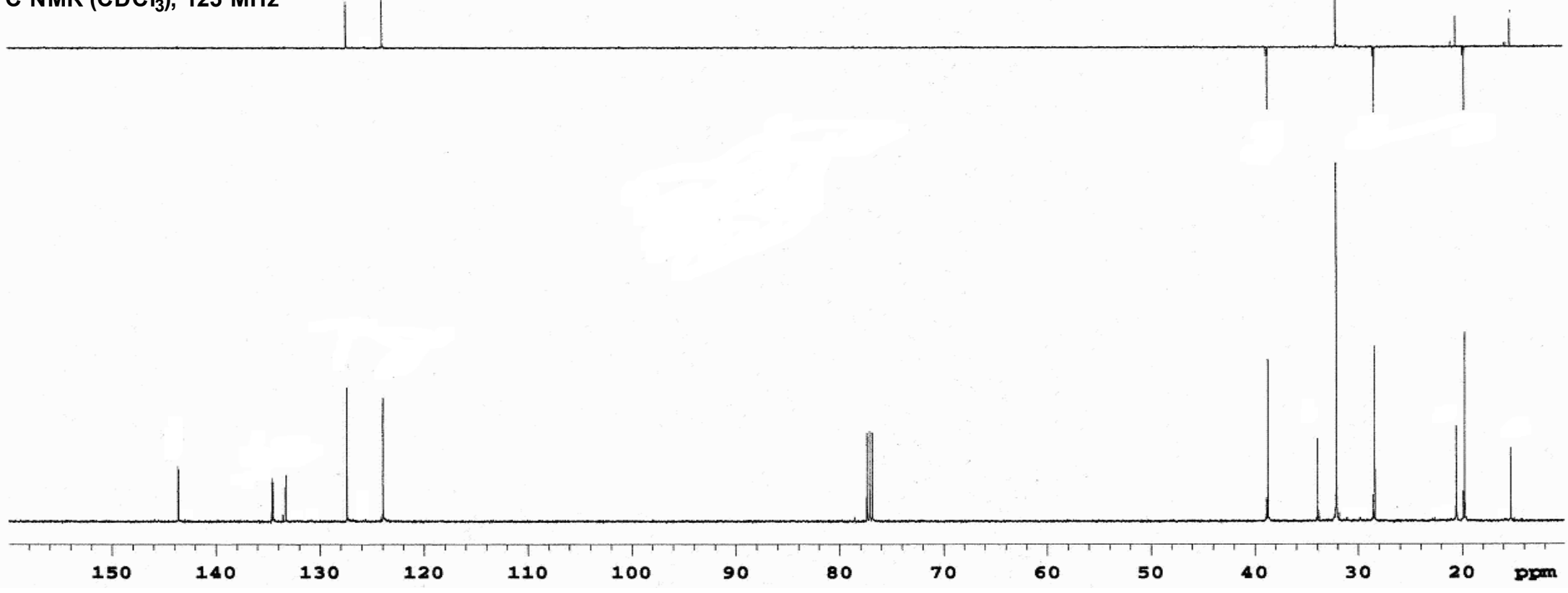
HMQC

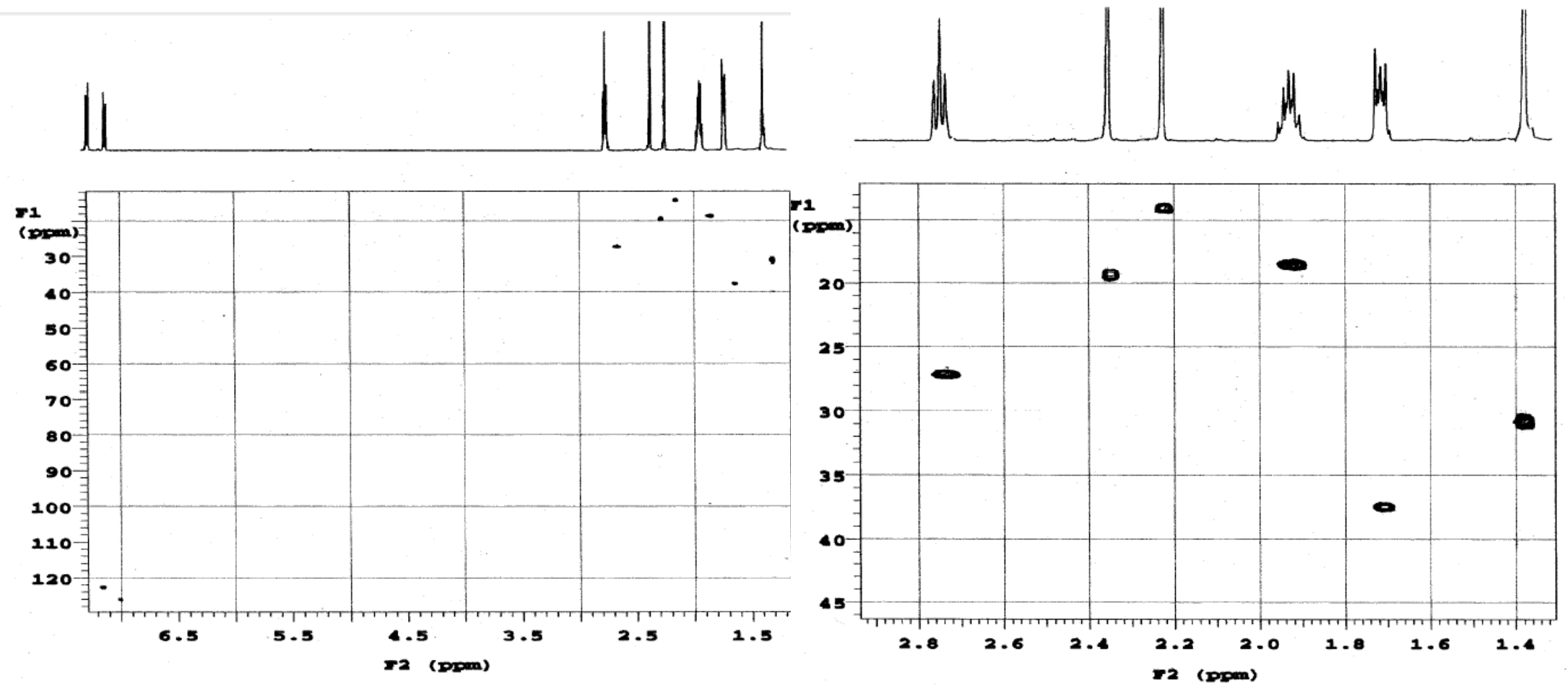


HMBC
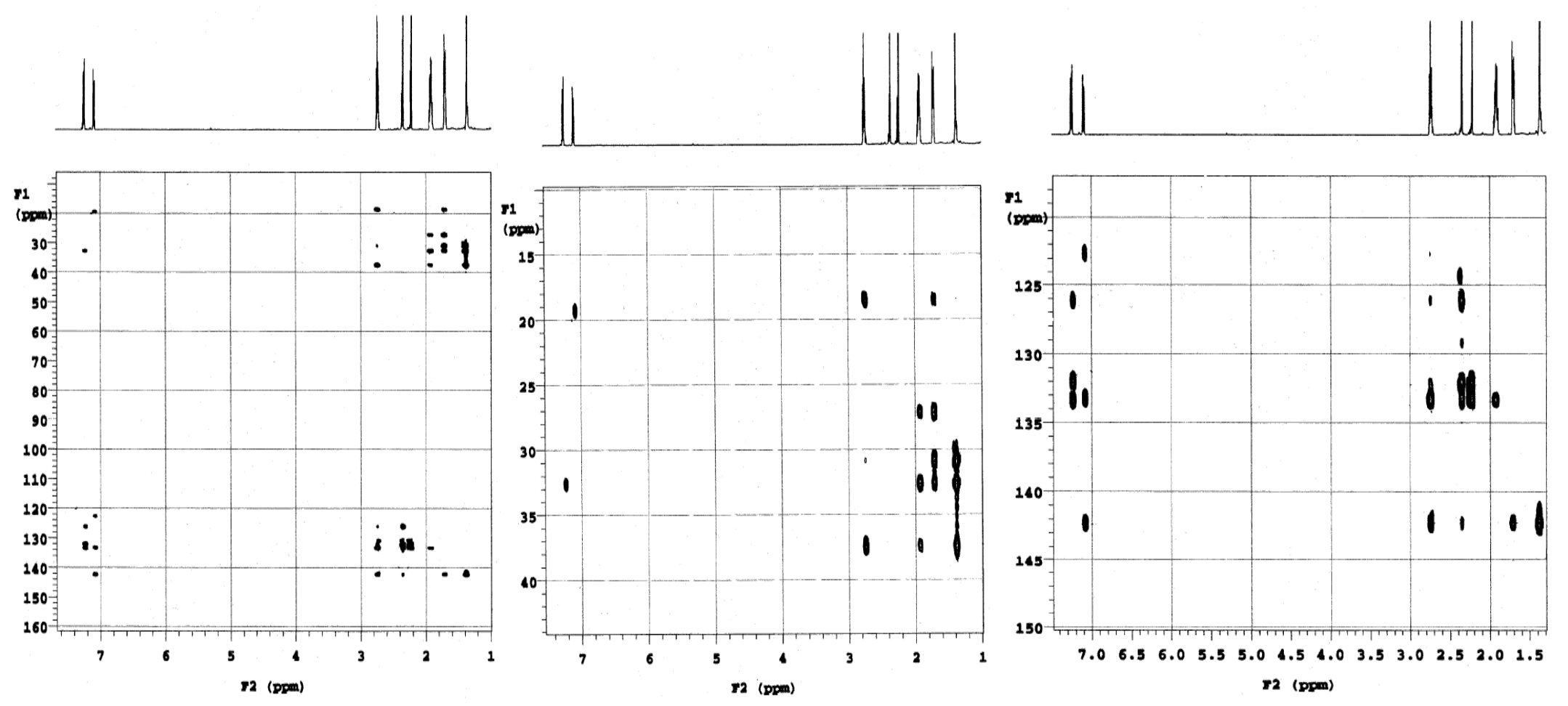


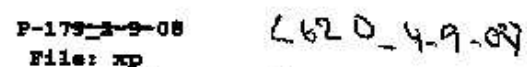
simple 1di traptady

Ful =e: Anquenoe: Mput

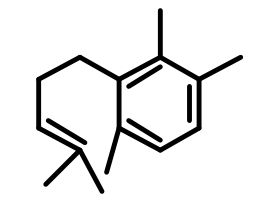

4

${ }^{1} \mathrm{H}$ NMR $\left(\mathrm{CDCl}_{3}\right), 500 \mathrm{MHz}$

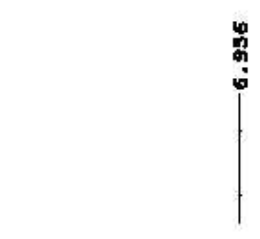

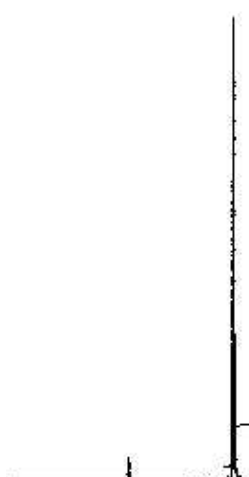
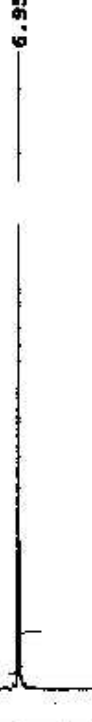

7

3.20

6

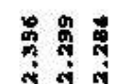

i)

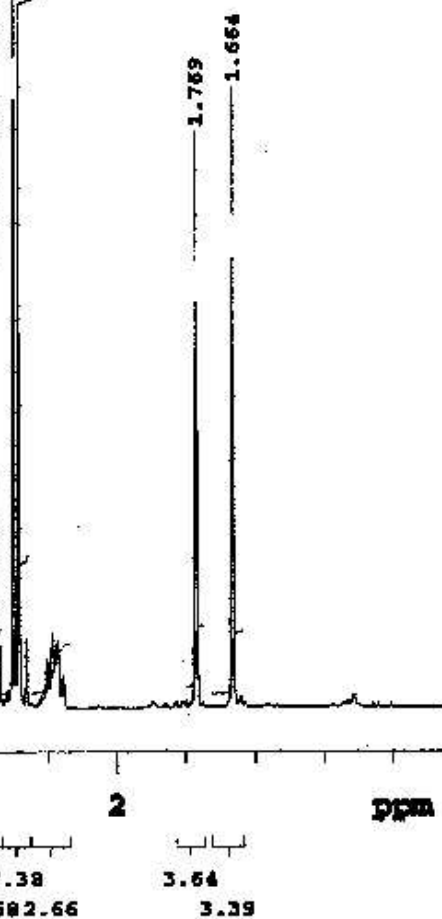




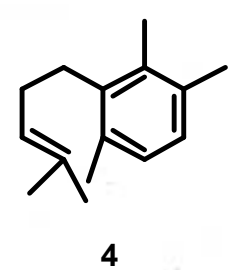

${ }^{13} \mathrm{C} \mathrm{NMR}\left(\mathrm{CDCl}_{3}\right), 125 \mathrm{MHz}$

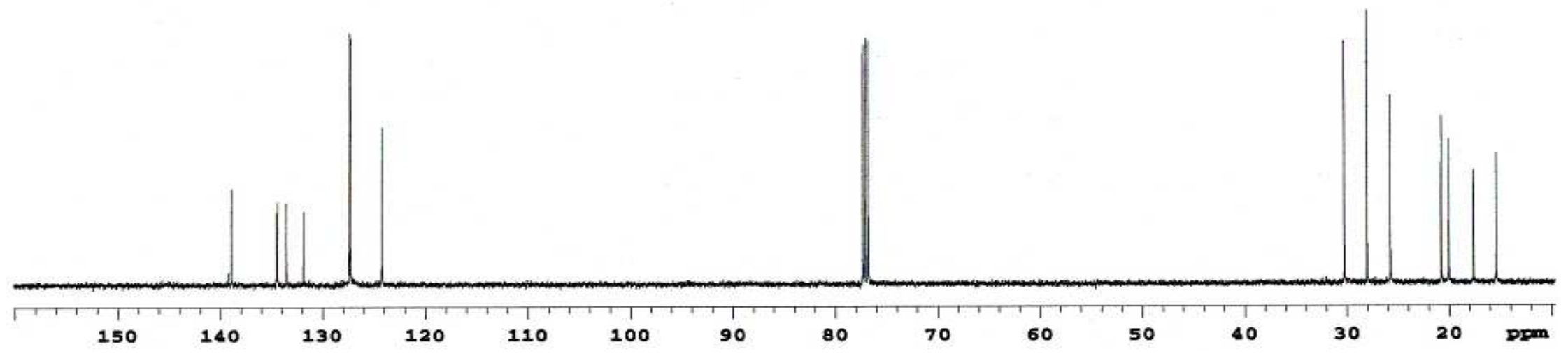


HMQC
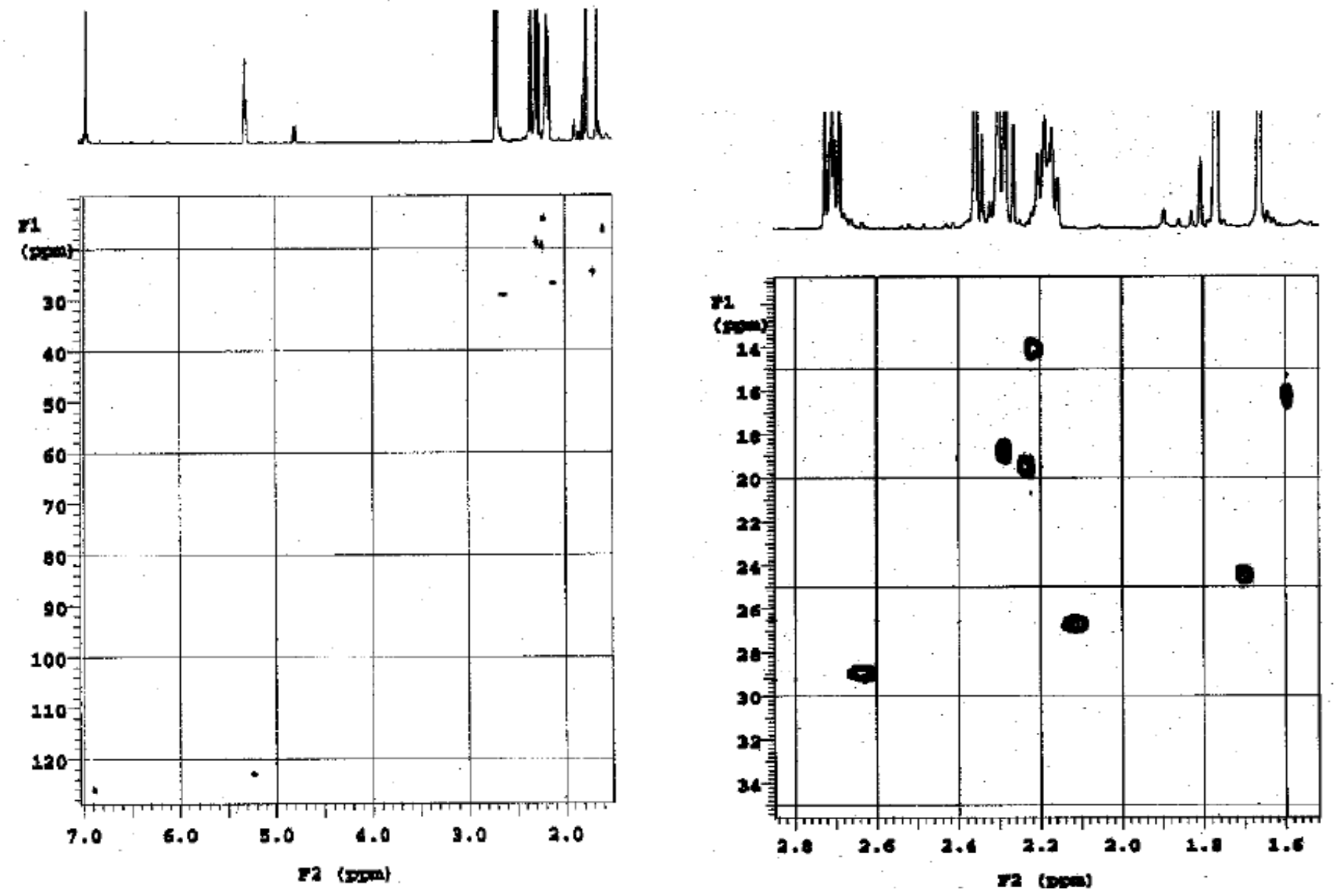
HMBC
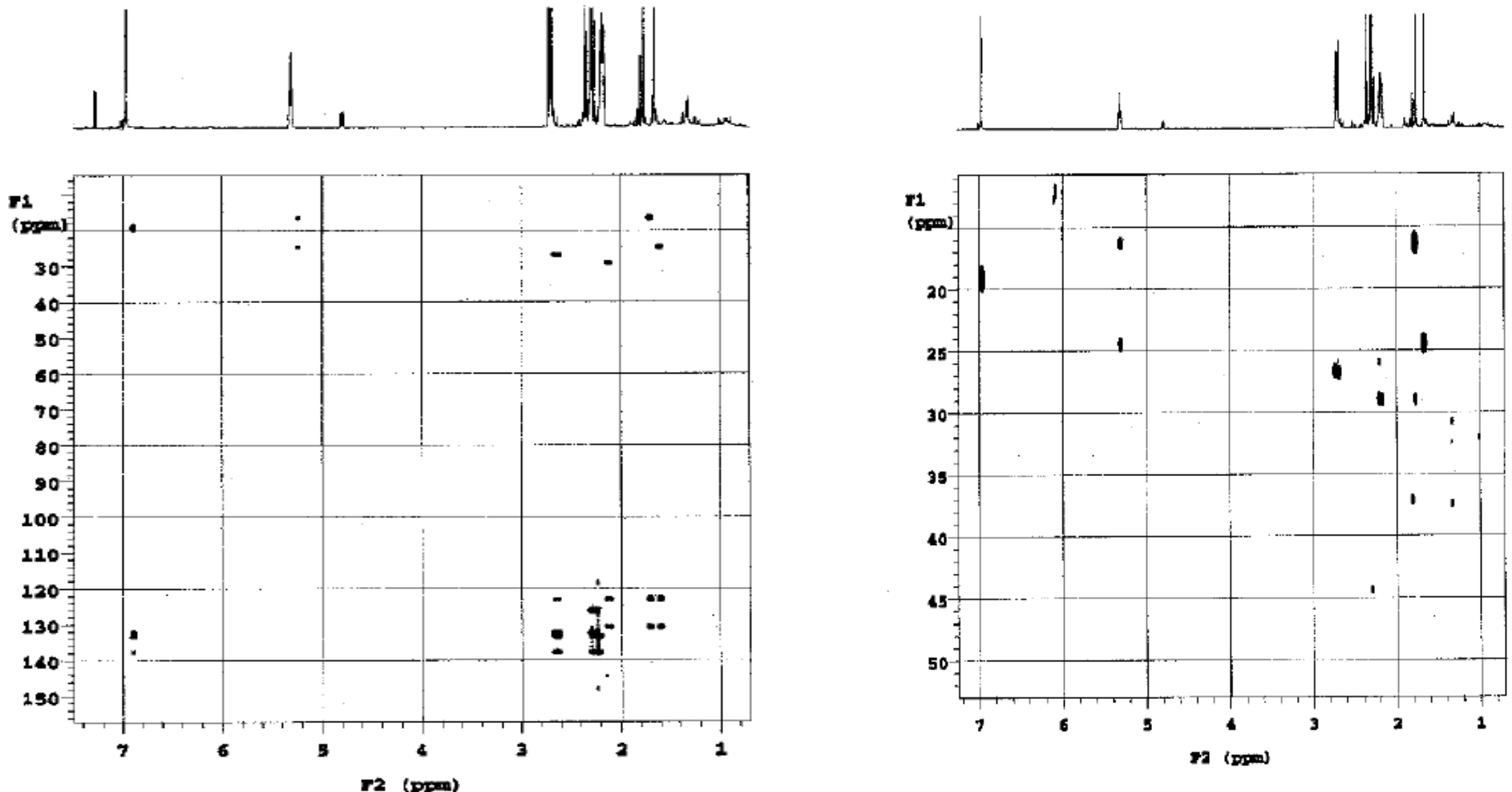
HMBC
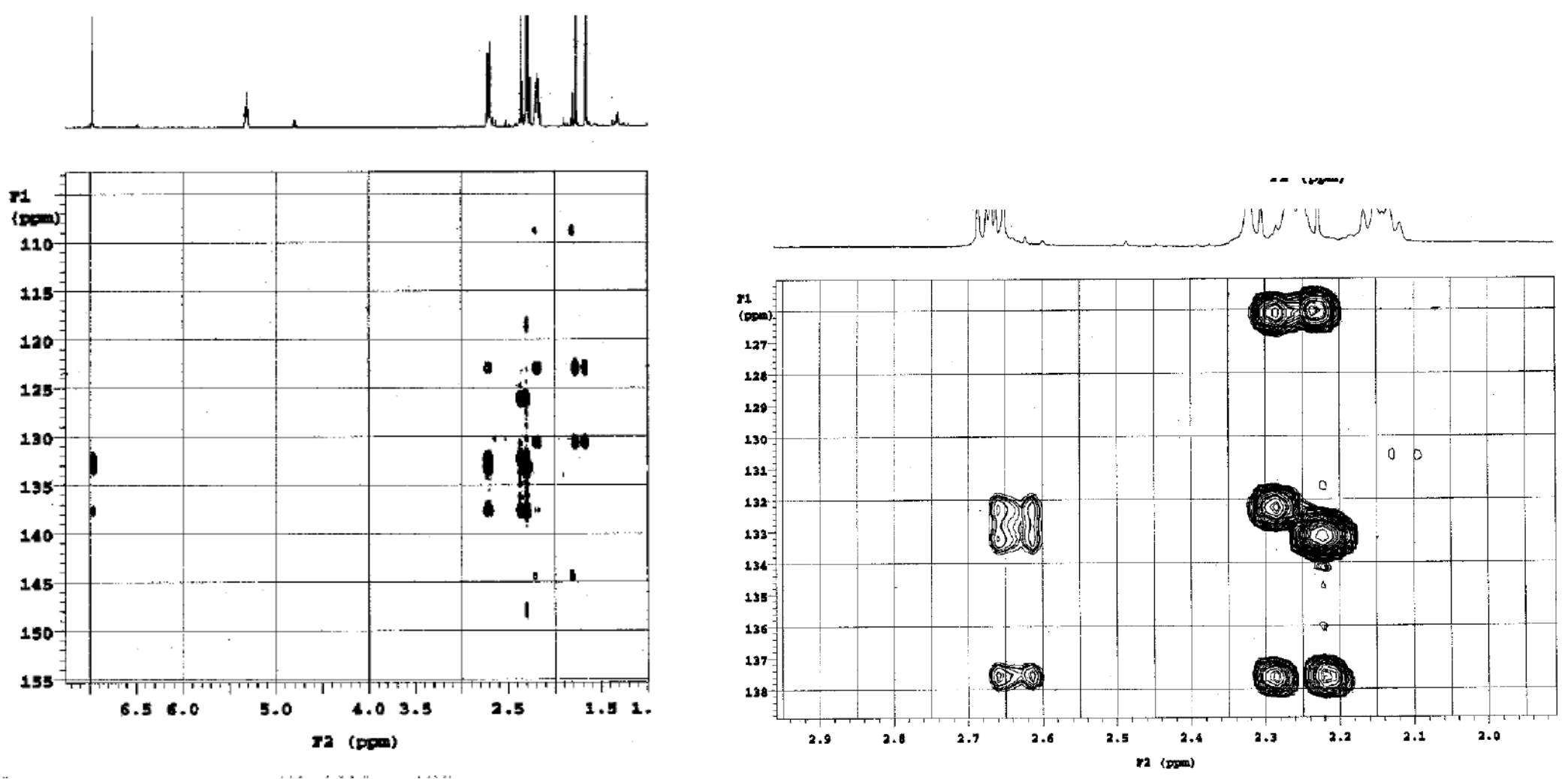
NOE
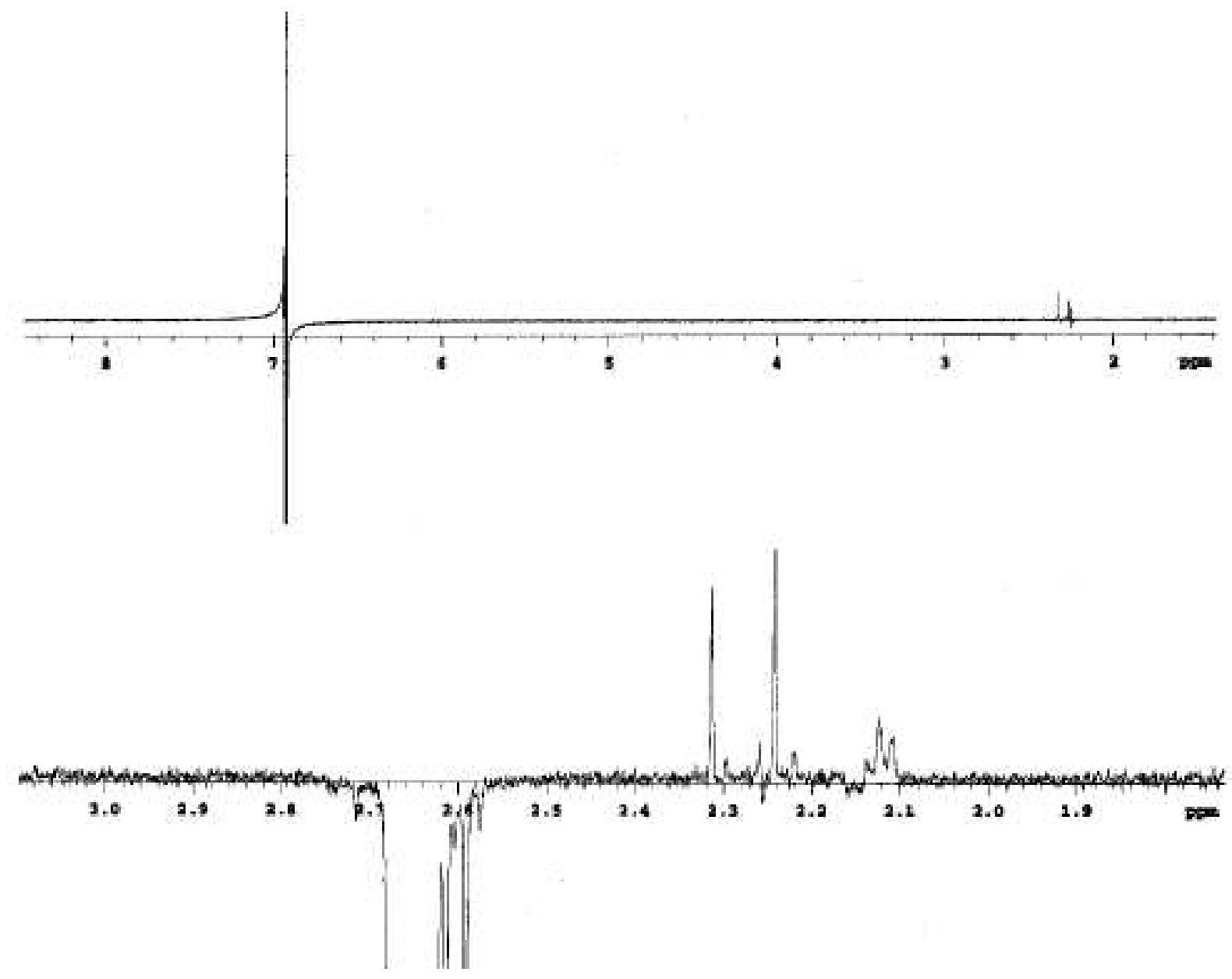


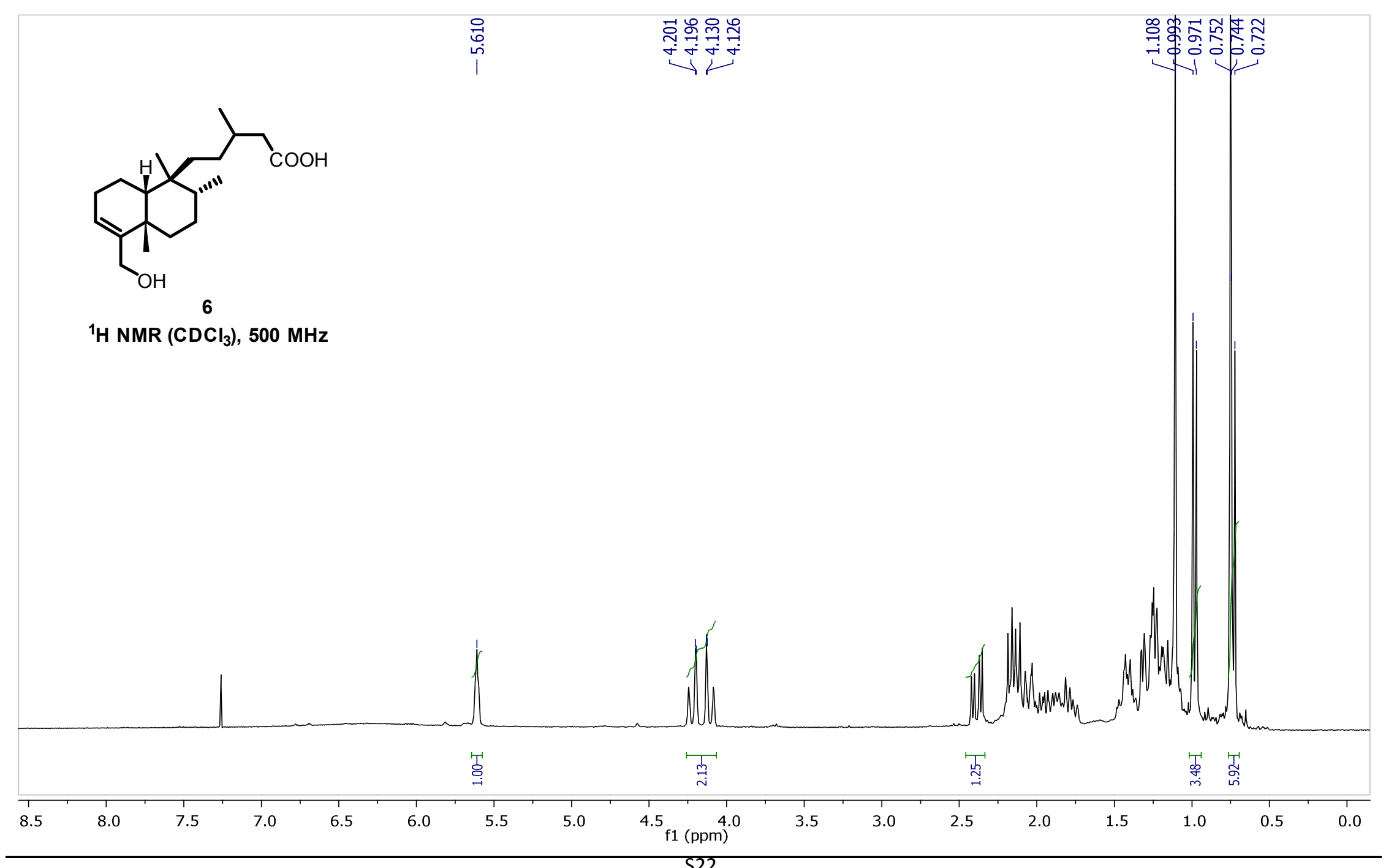

S22 

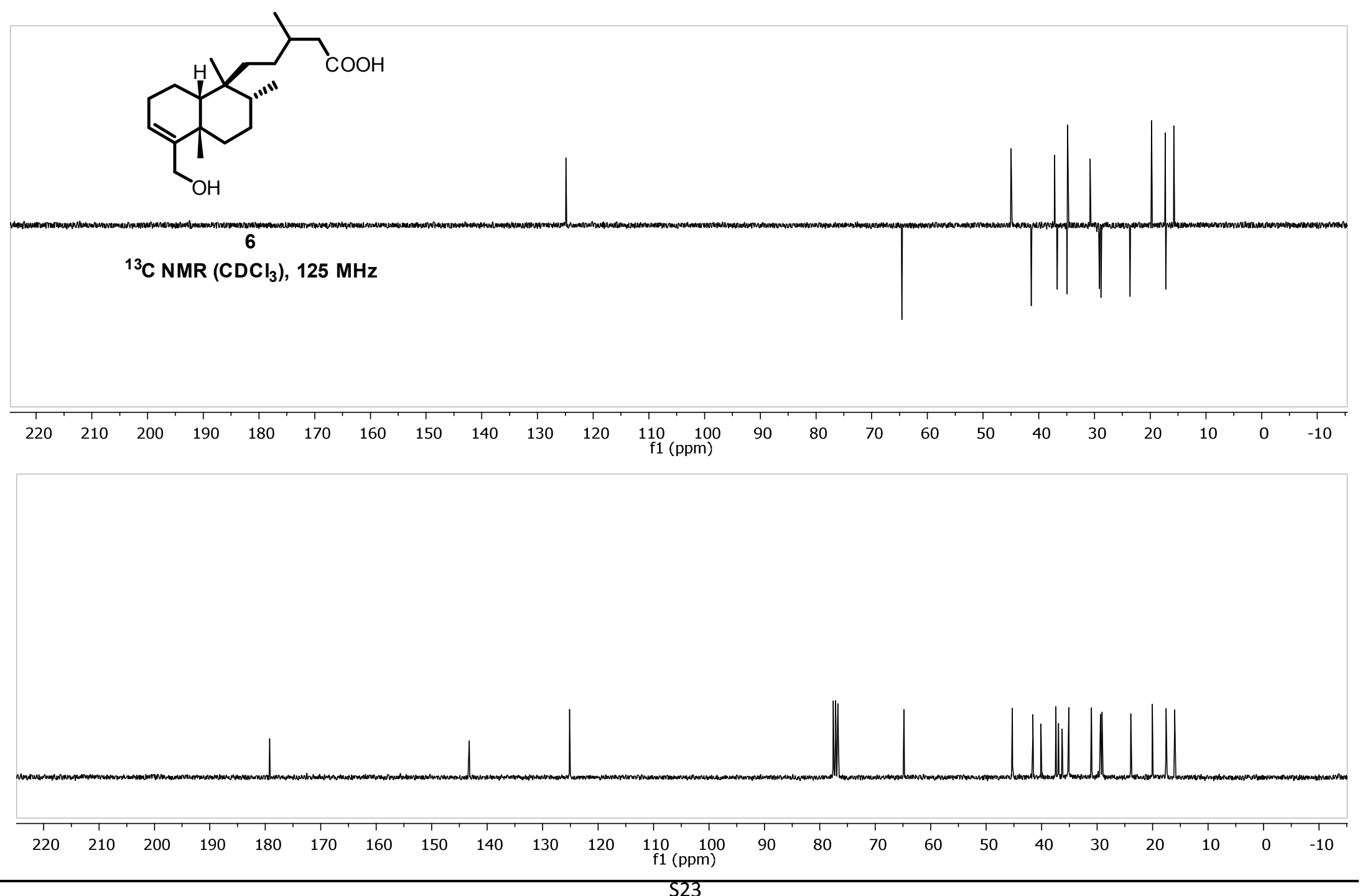
1H-CON-525-B-36

CON-525-B-36

${ }^{1} \mathrm{H}$ NMR $\left(\mathrm{CDCl}_{3}\right), 500 \mathrm{MHz}$

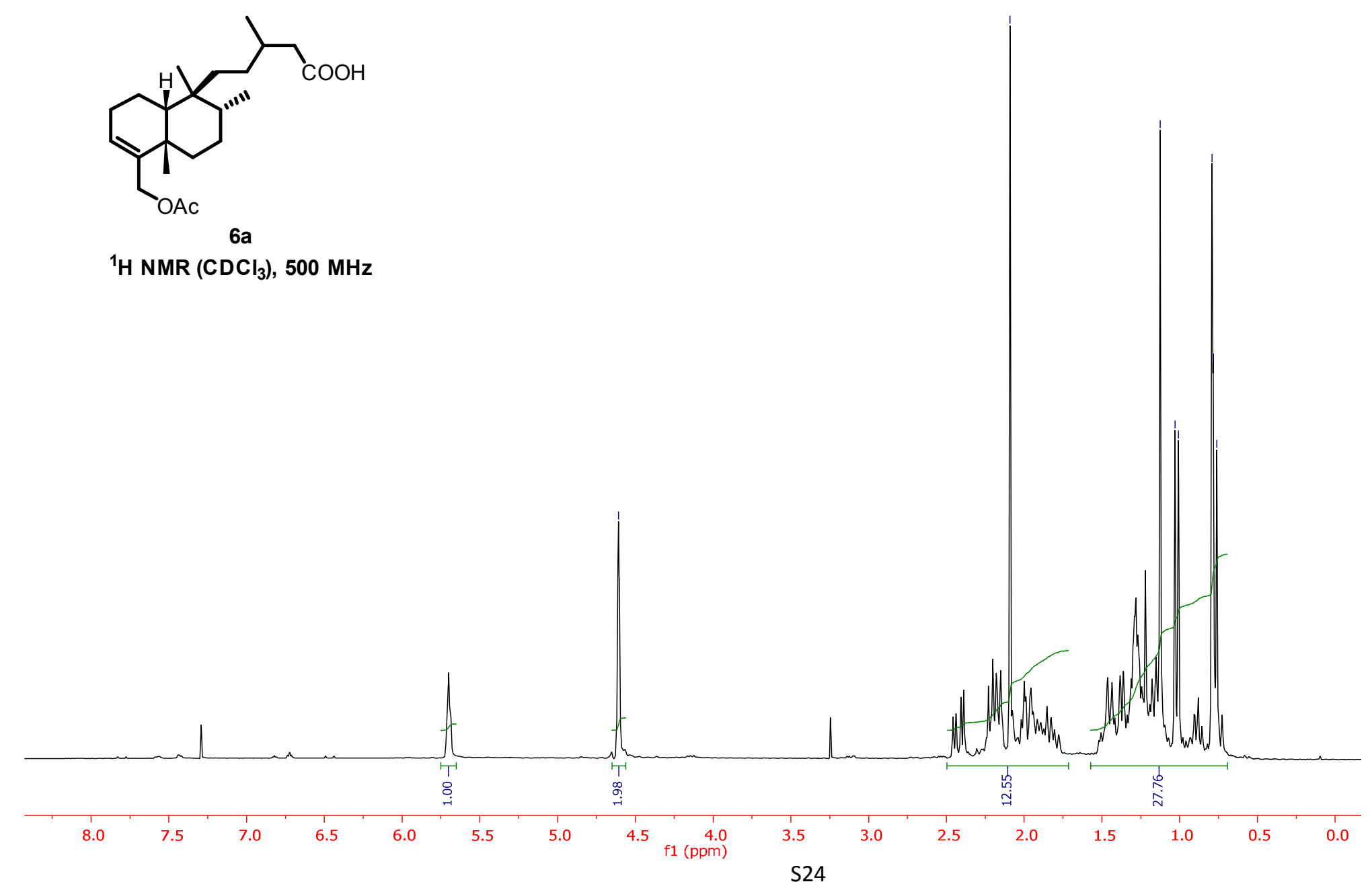

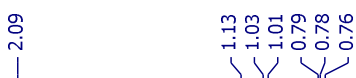




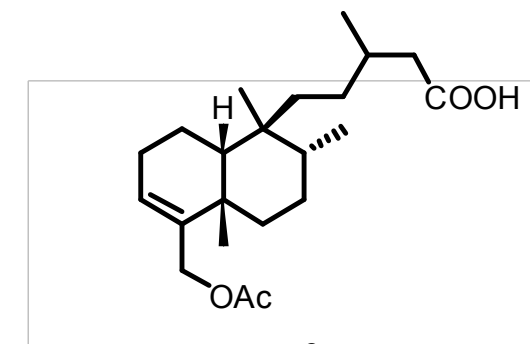

$6 a$

${ }^{13} \mathrm{C}$ NMR $\left(\mathrm{CDCl}_{3}\right), 125 \mathrm{MHz}$

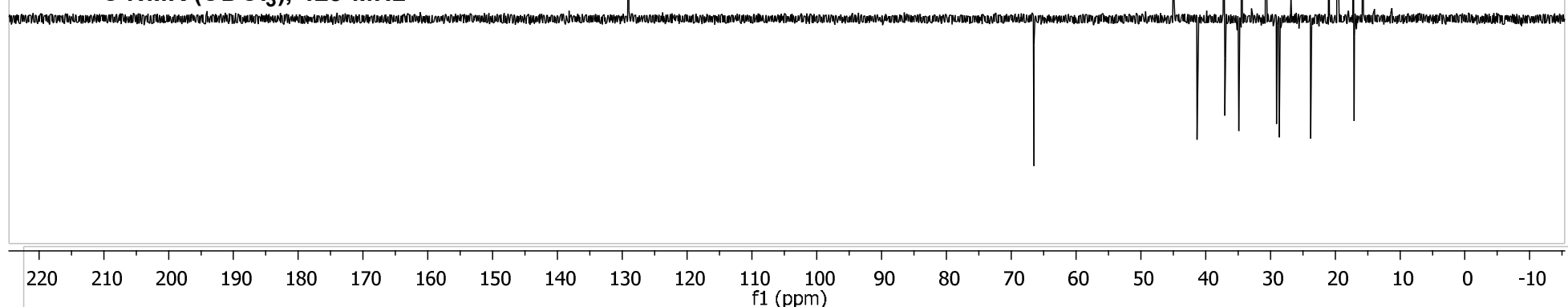

$\begin{array}{llllllllllll}220 & 210 & 200 & 190 & 180 & 170 & 160 & 150 & 140 & 130 & 120 & 110 \\ \mathrm{f} 1(\mathrm{ppm})\end{array}$
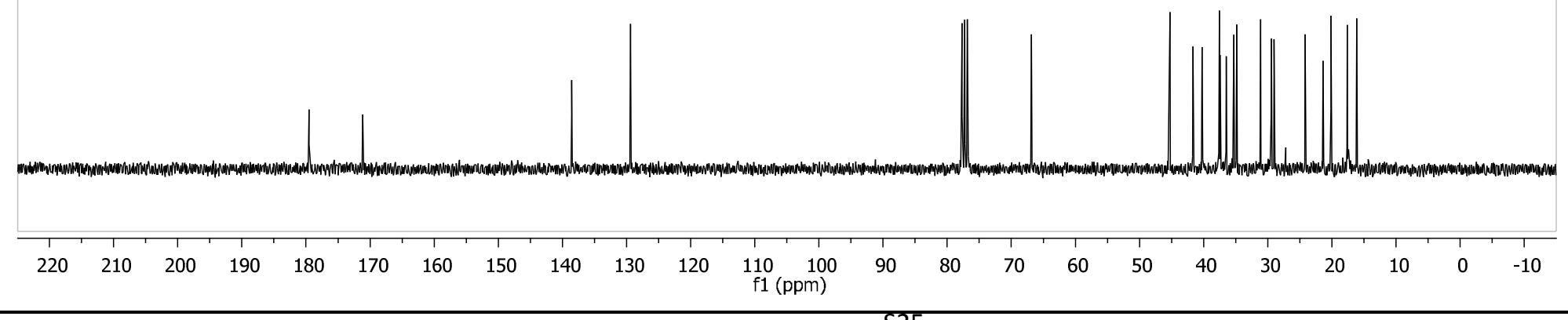


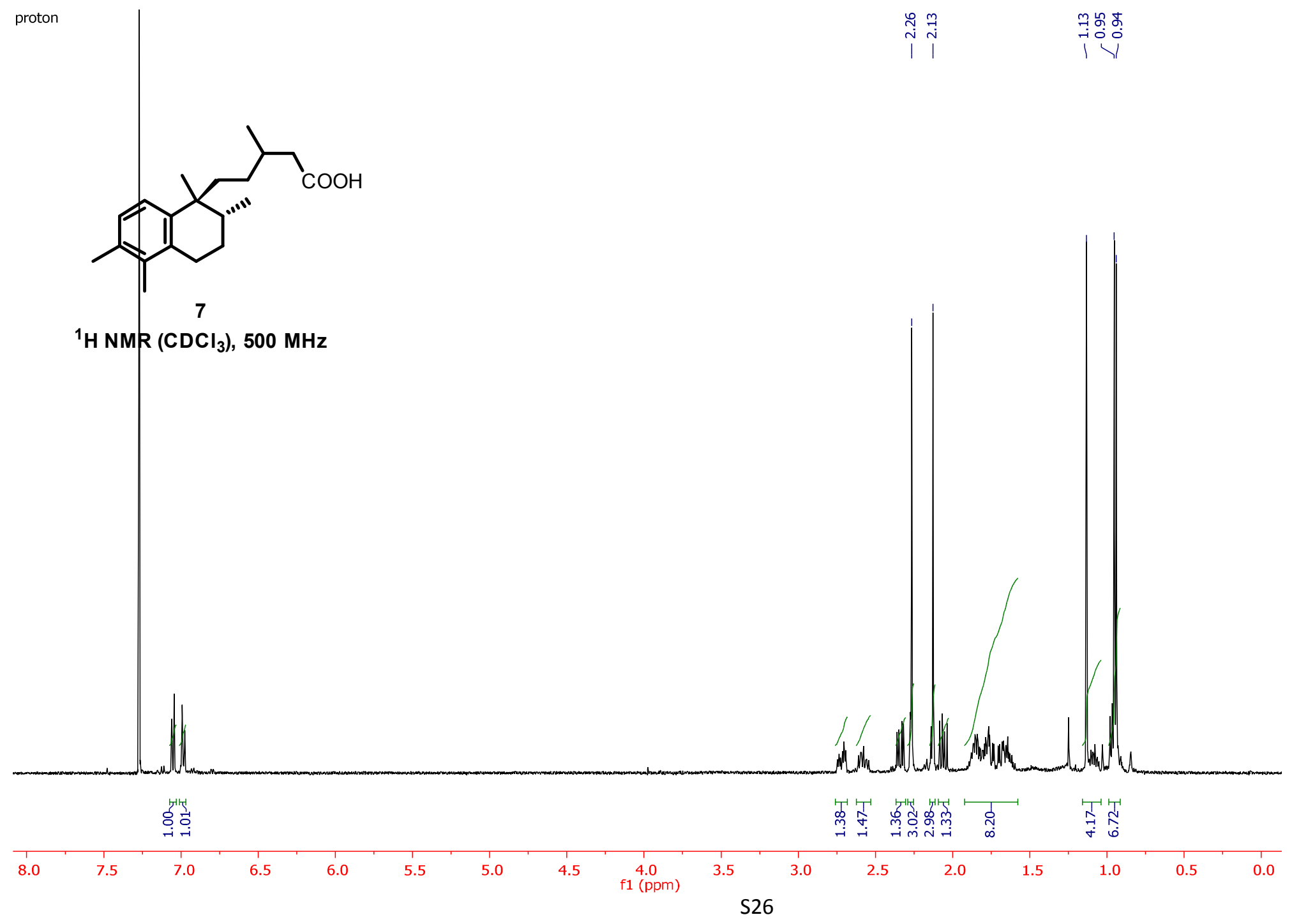




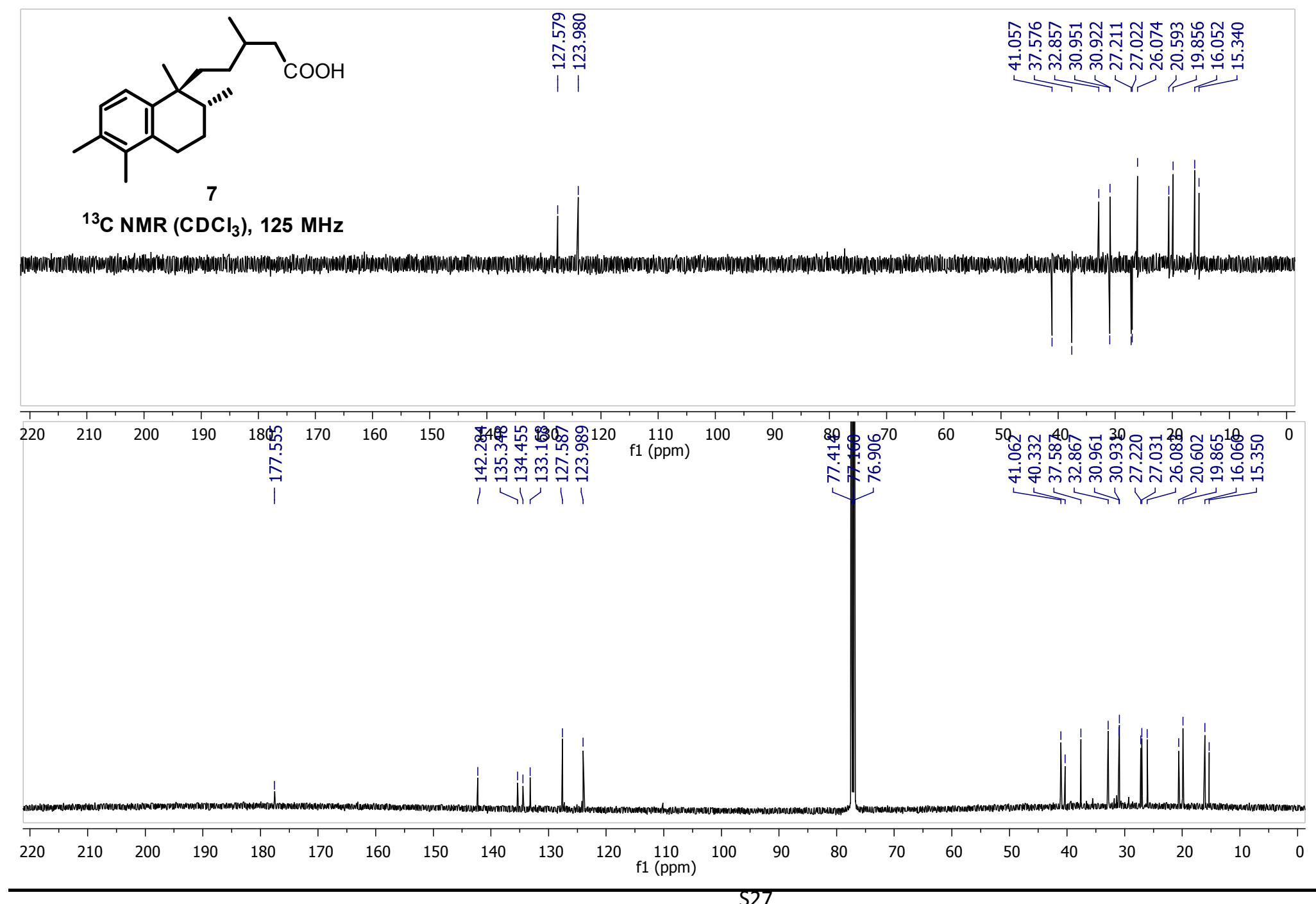




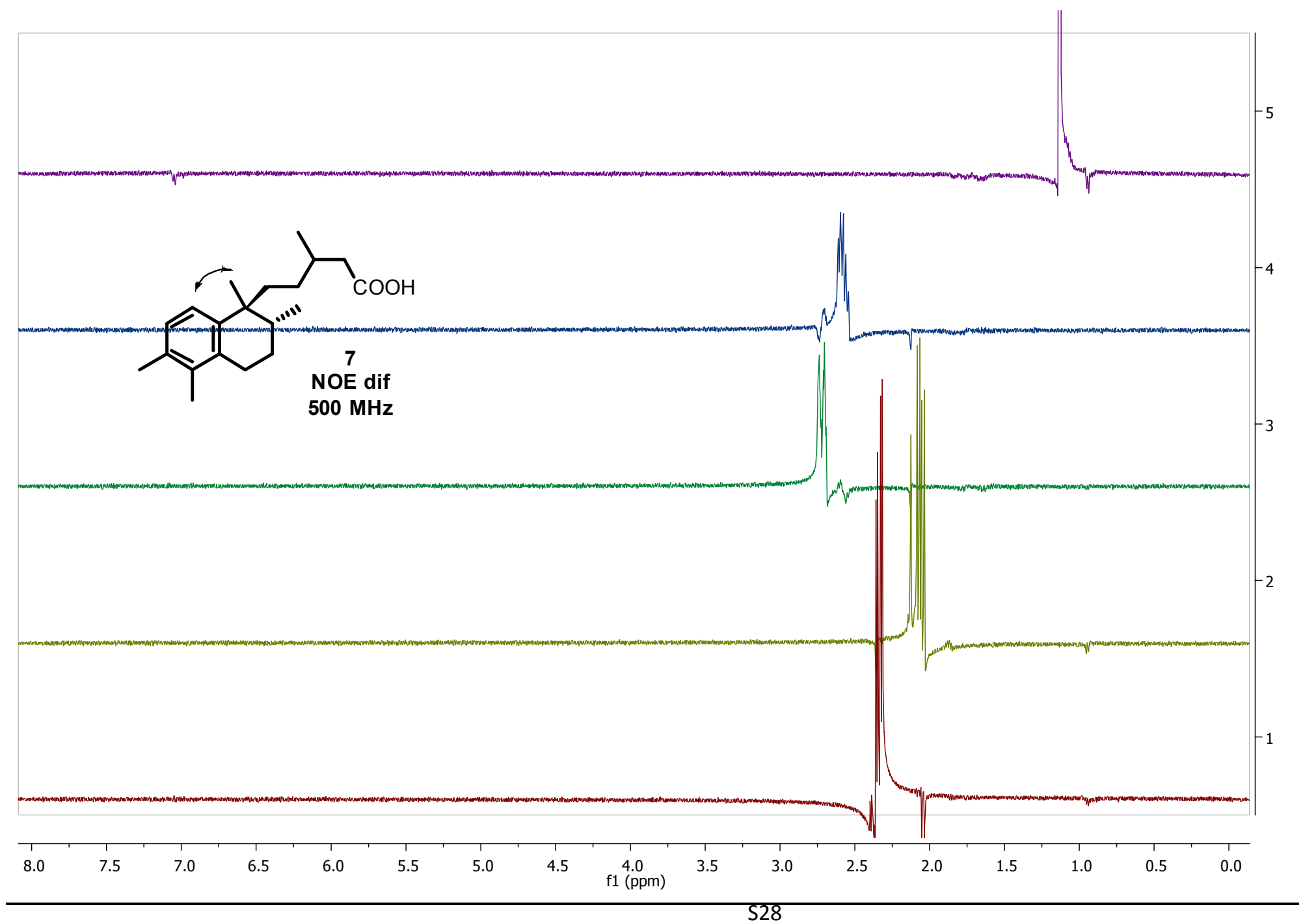




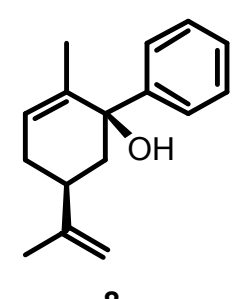

${ }^{1} \mathrm{H} \mathrm{NMR}\left(\mathrm{CDCl}_{3}\right), 400 \mathrm{MHz}$

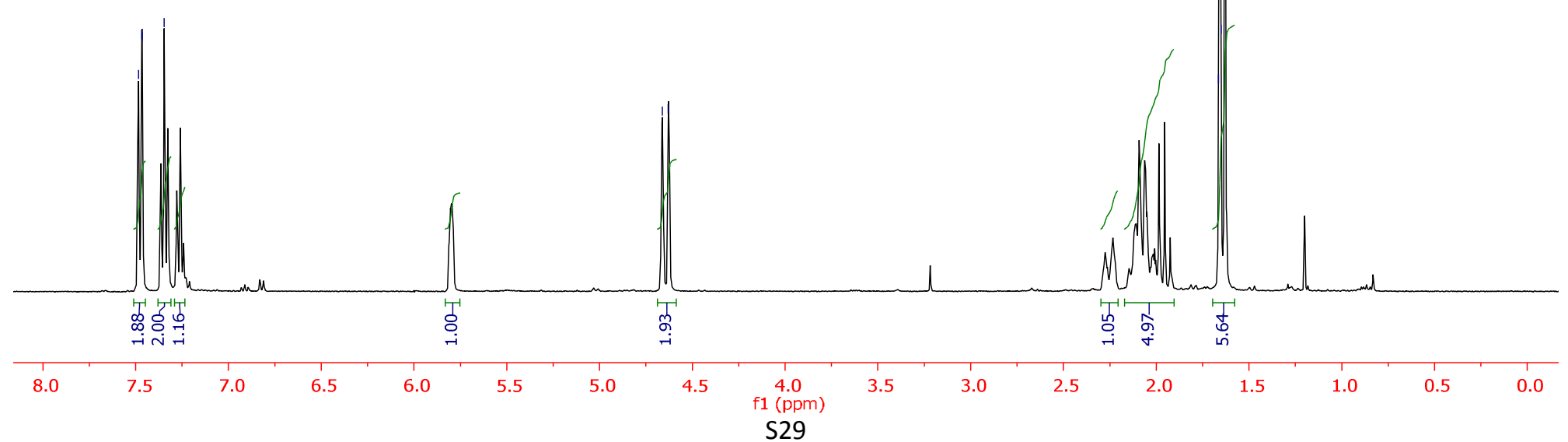




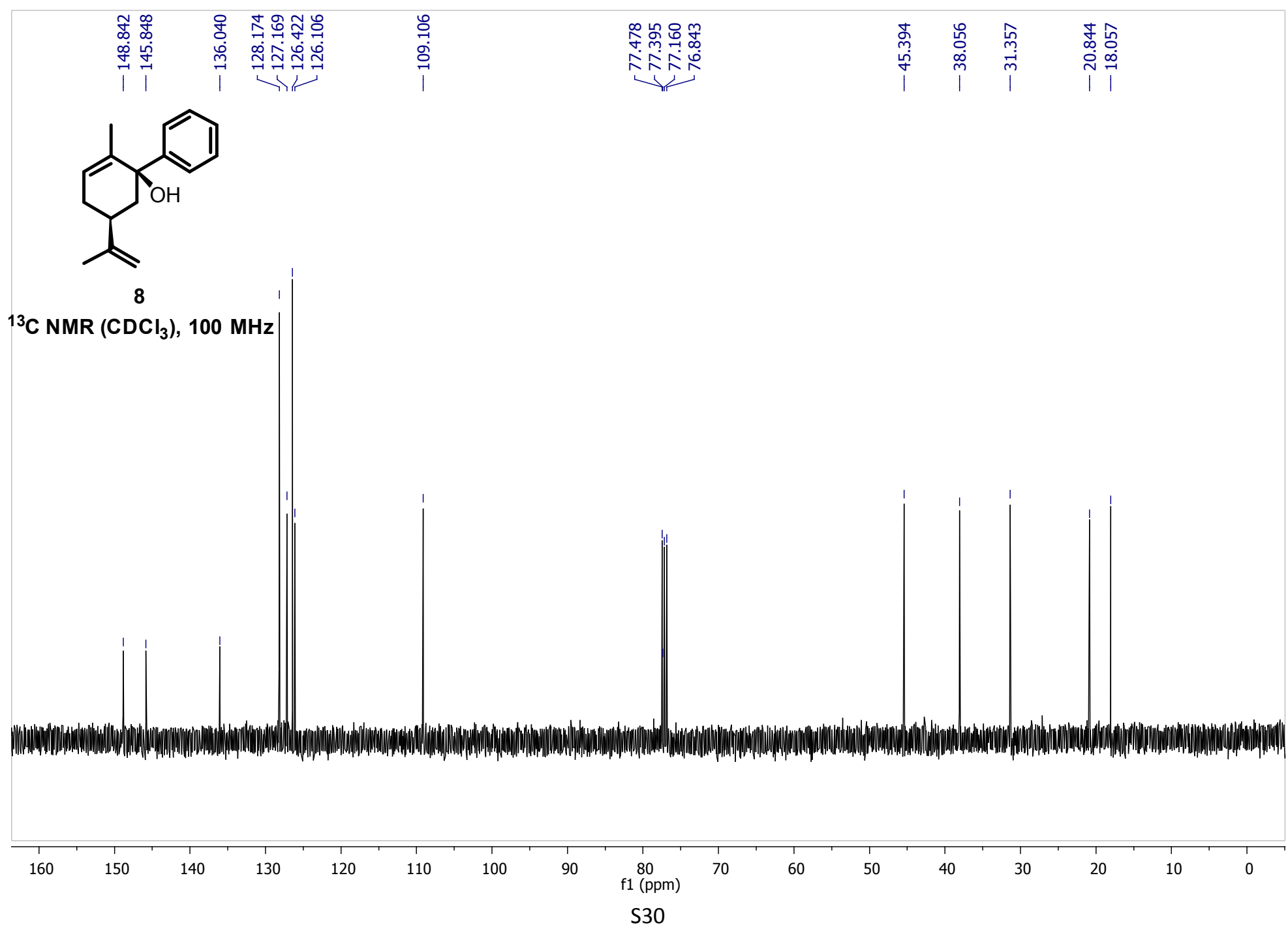




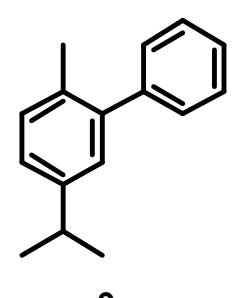

${ }^{1} \mathrm{H}$ NMR $\left(\mathrm{CDCl}_{3}\right), 400 \mathrm{MHz}$

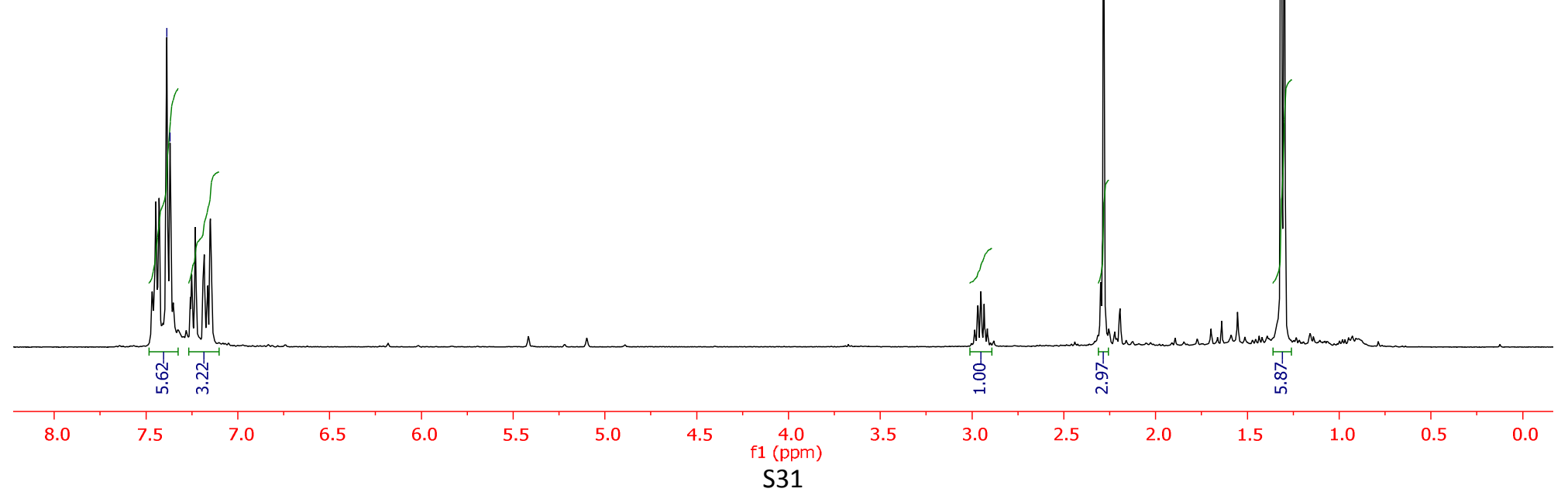




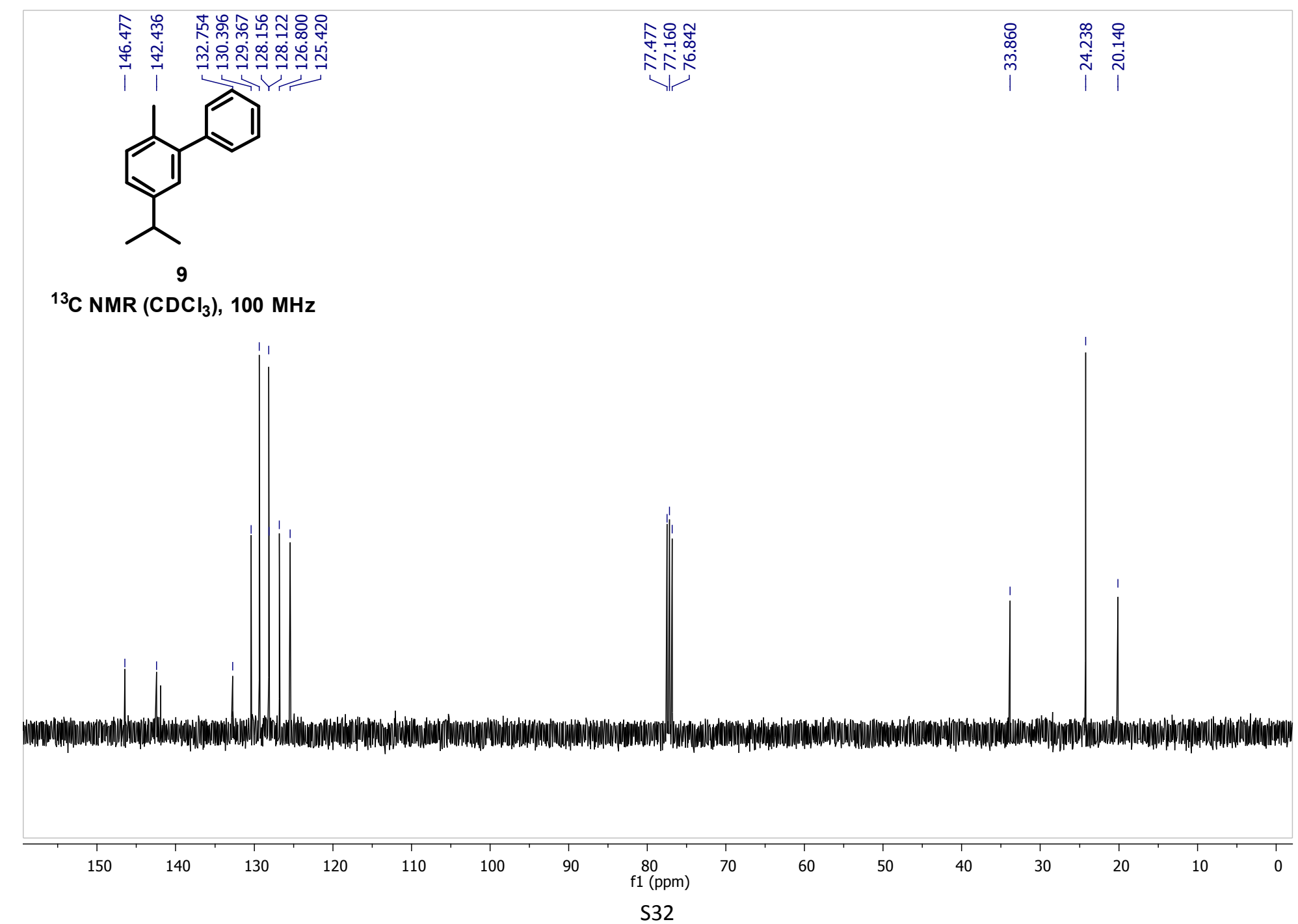




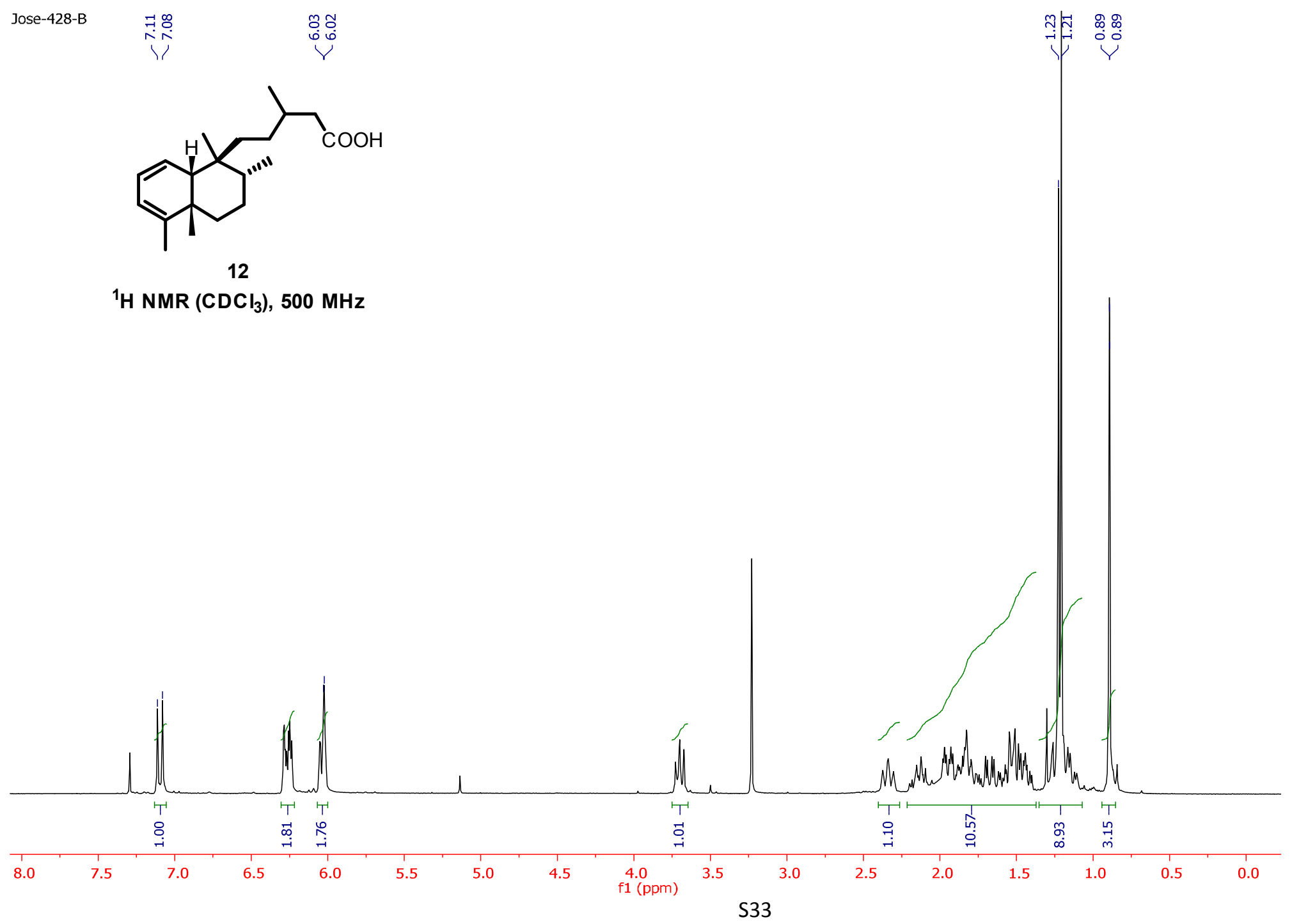




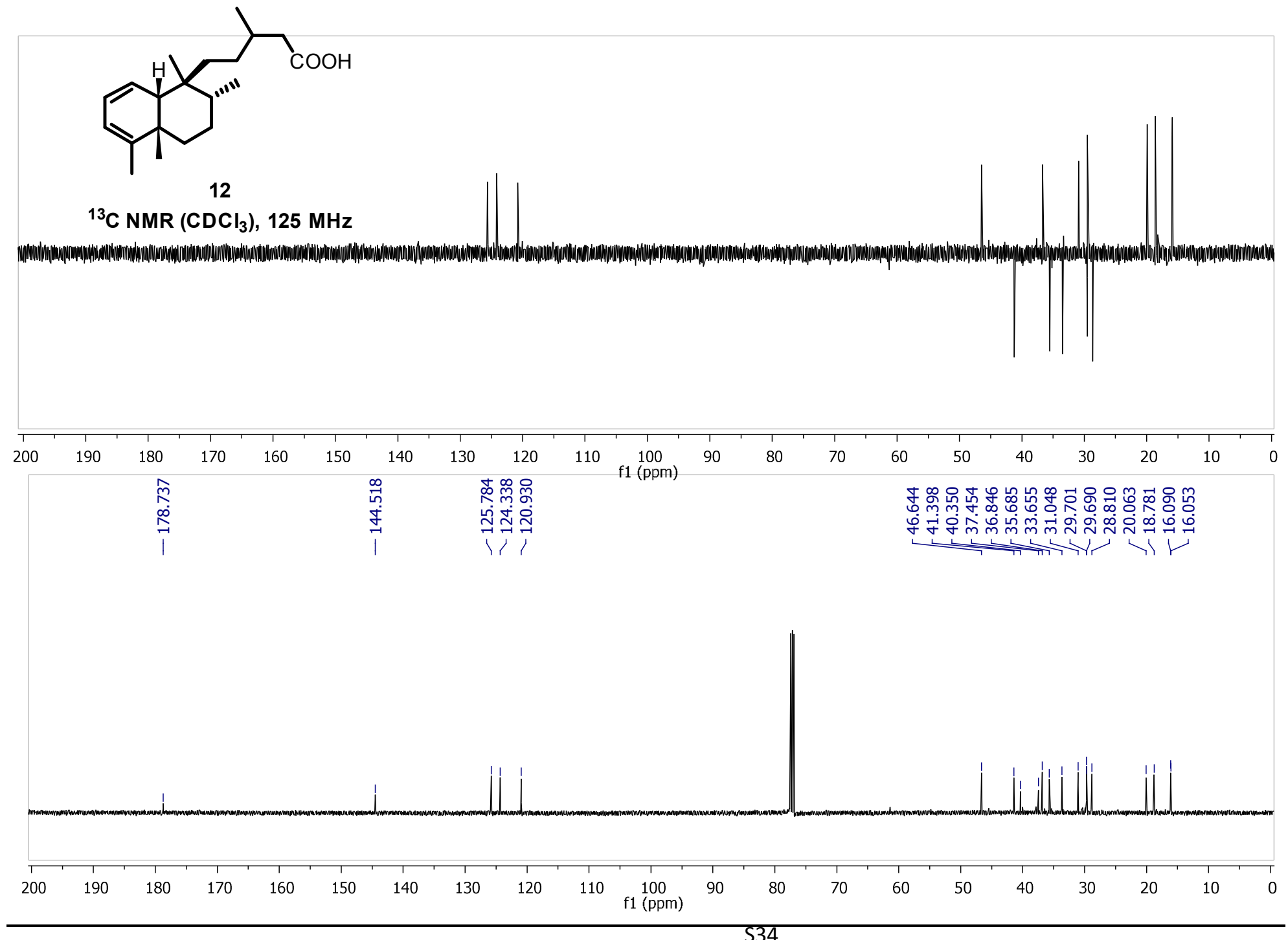

S34 


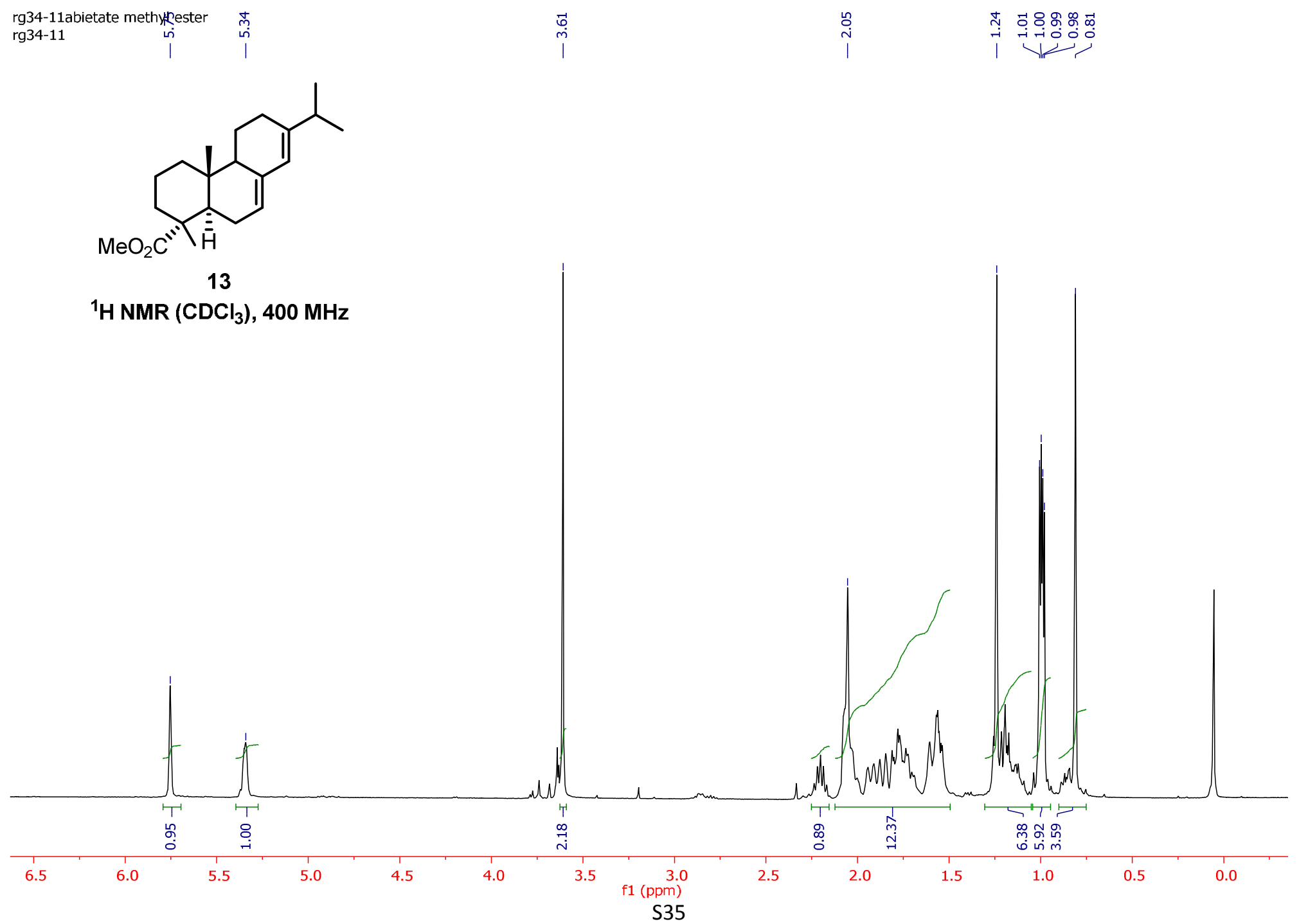




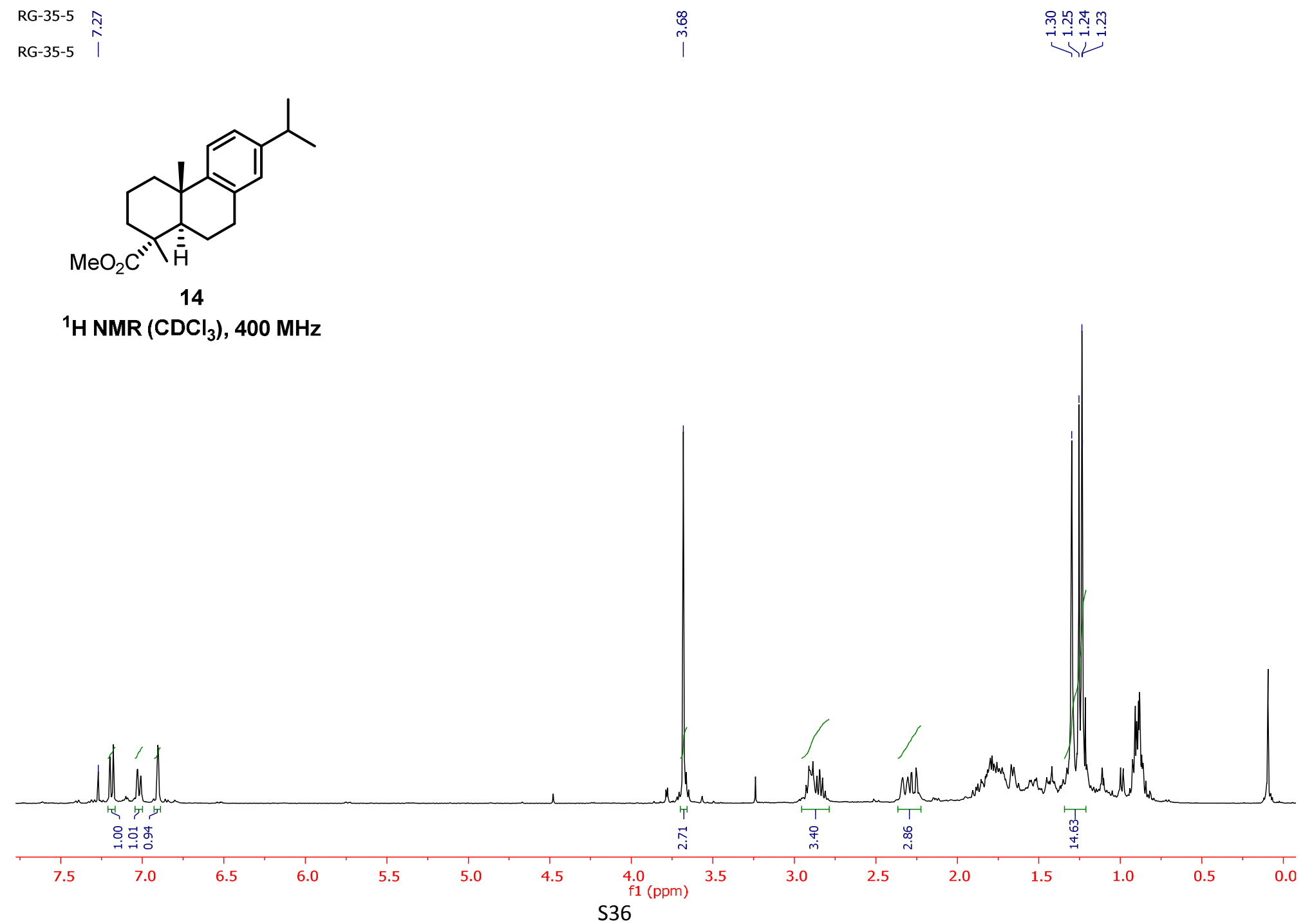




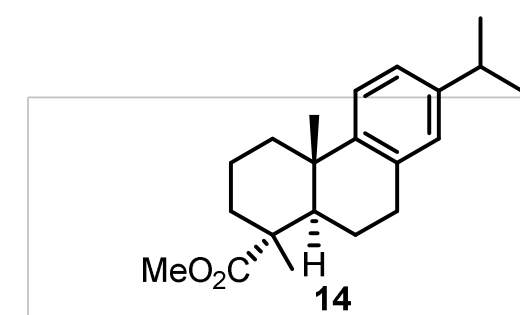

땡으용

워ㅁㅝㅝ

$\mathrm{MeO}_{2} \mathrm{C}$

${ }^{13} \mathrm{C} \mathrm{NMR}\left(\mathrm{CDCl}_{3}\right), 100 \mathrm{MHz}$

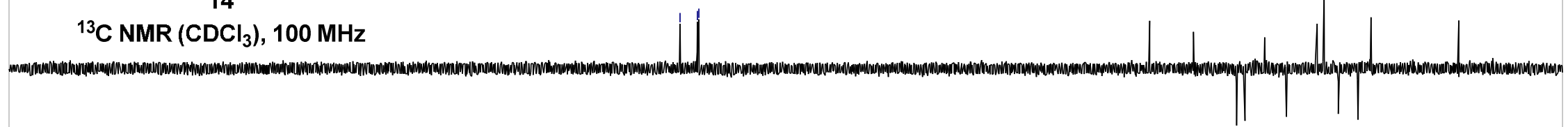

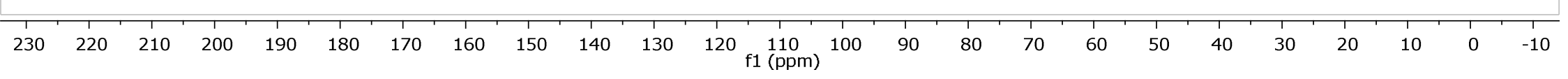

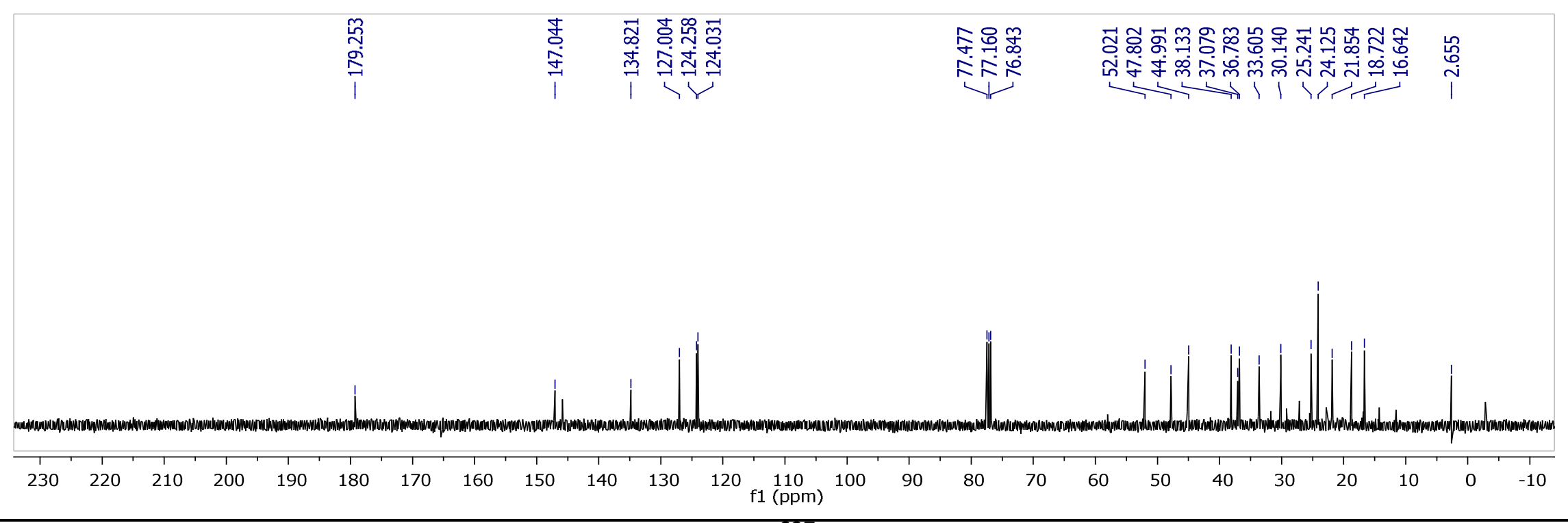




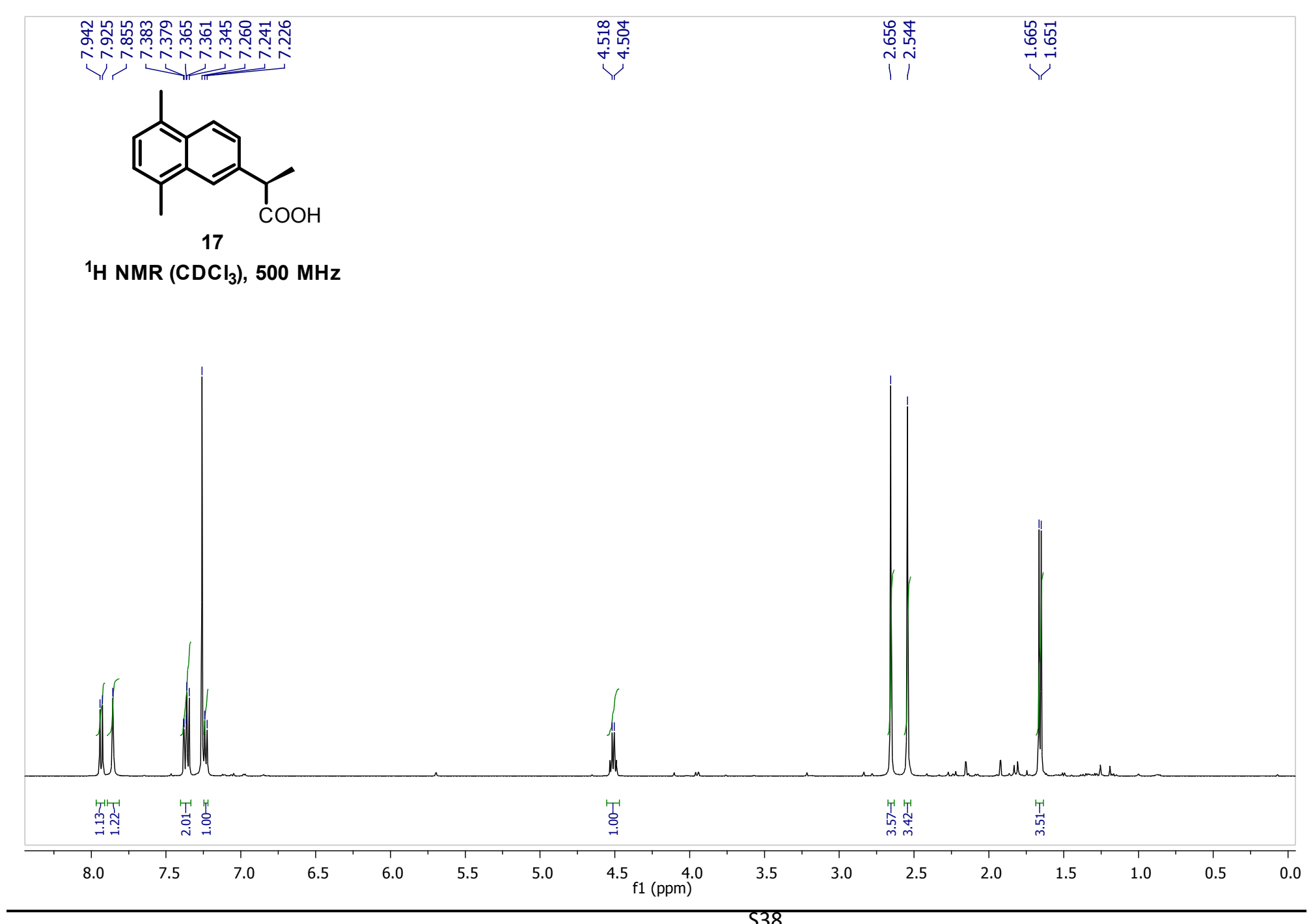




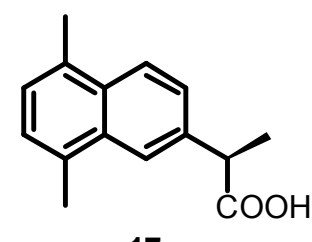

${ }^{13} \mathrm{C}$ NMR $\left(\mathrm{CDCl}_{3}\right), 125 \mathrm{MHz}$
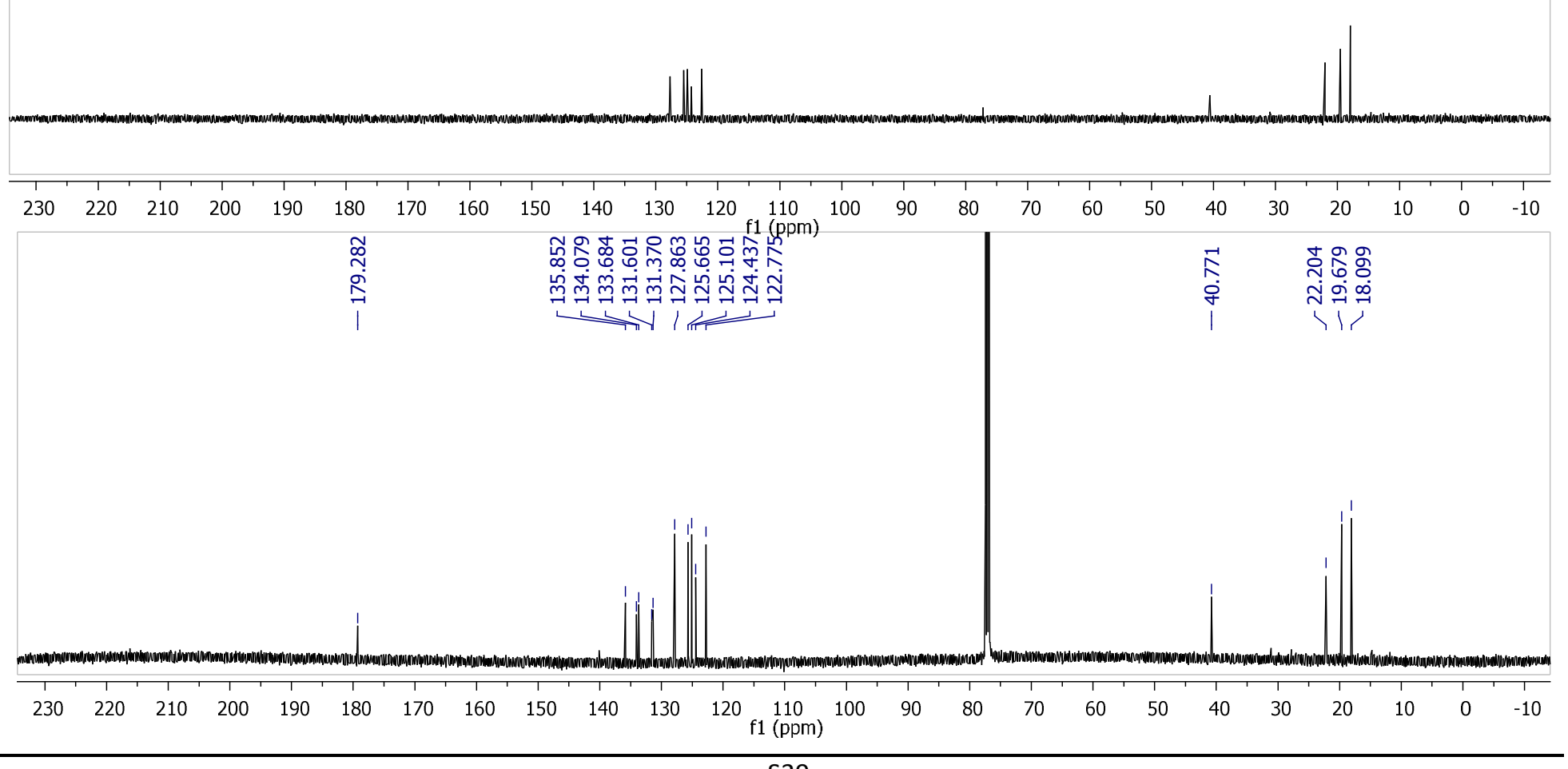


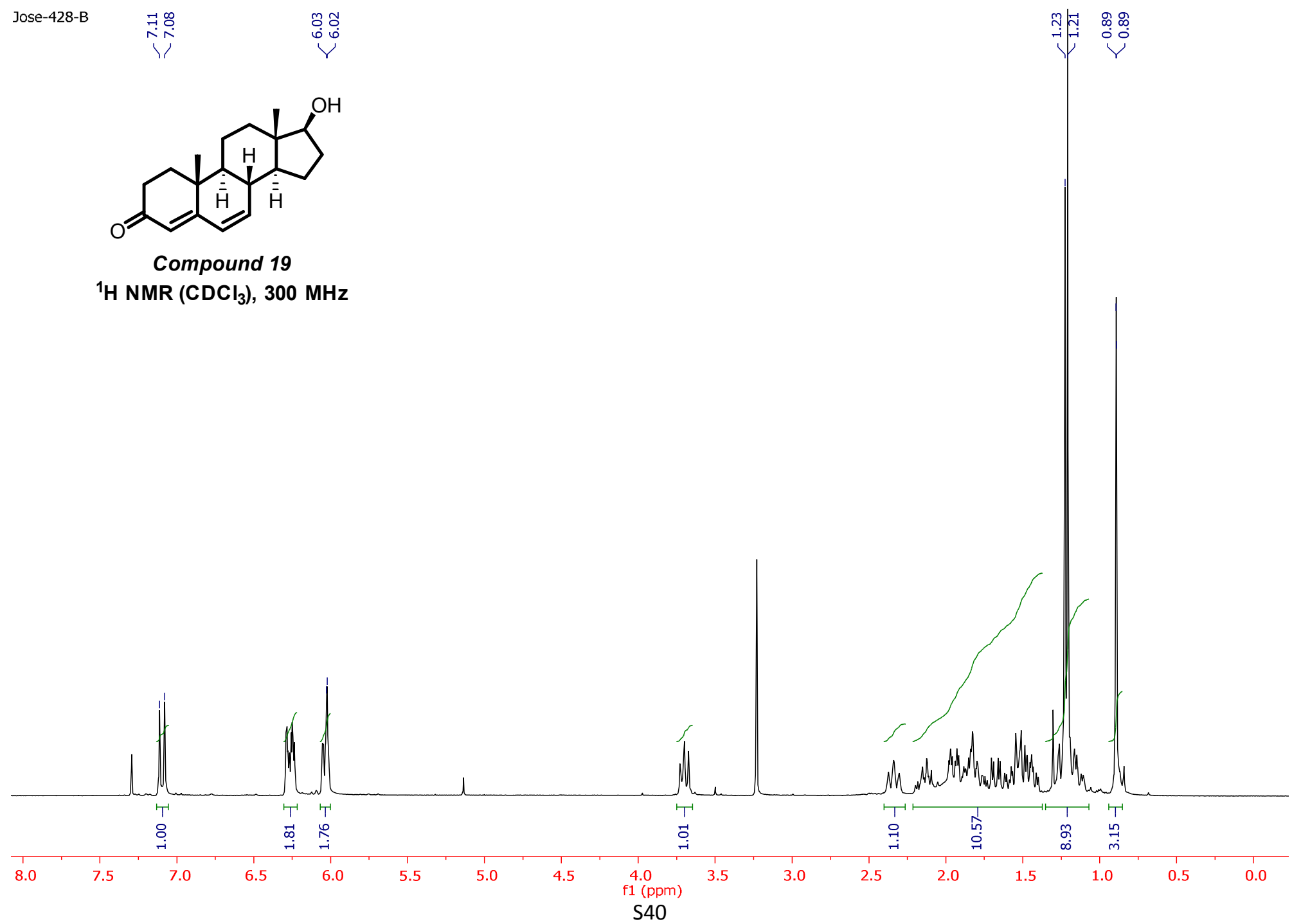



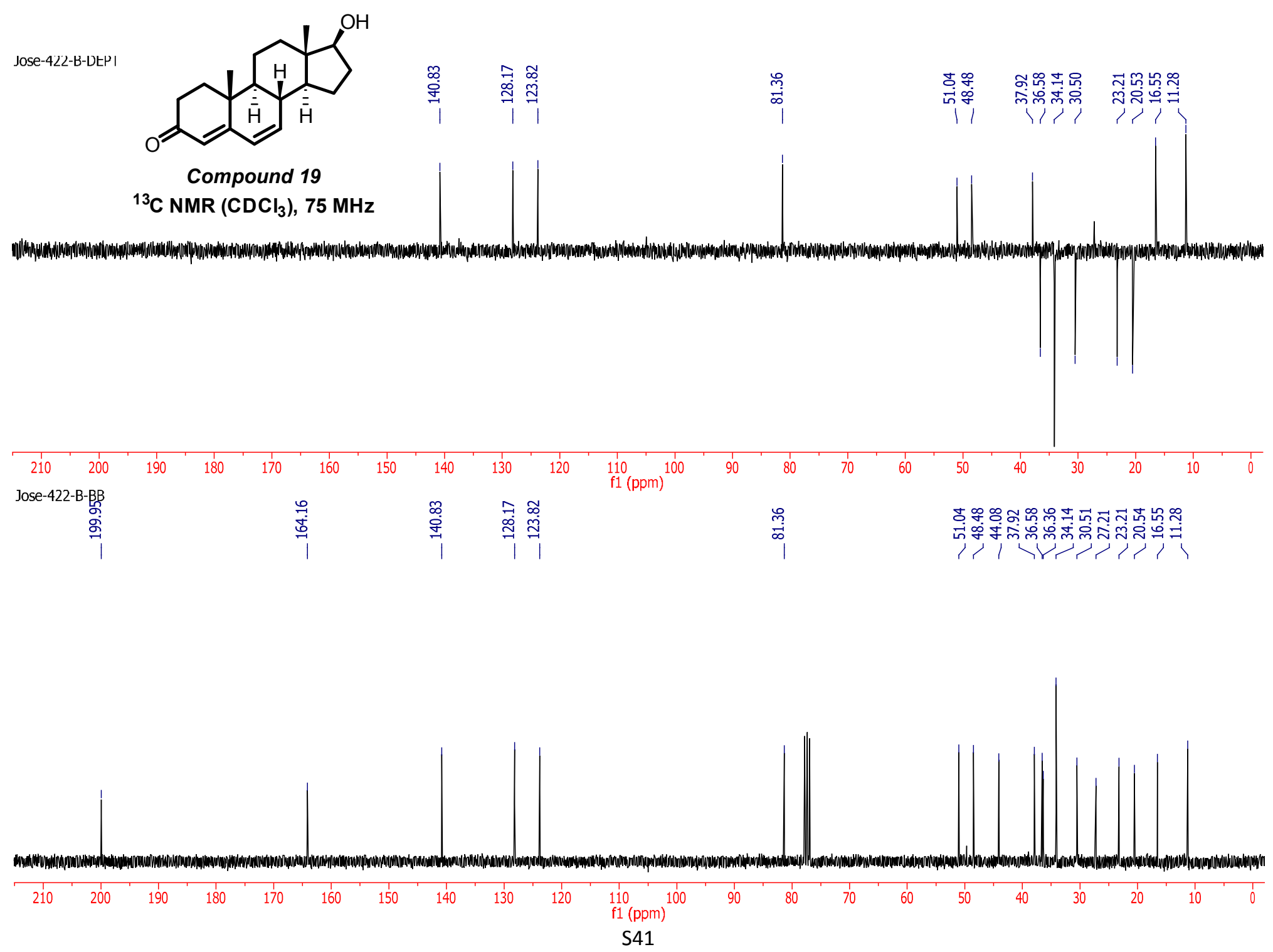


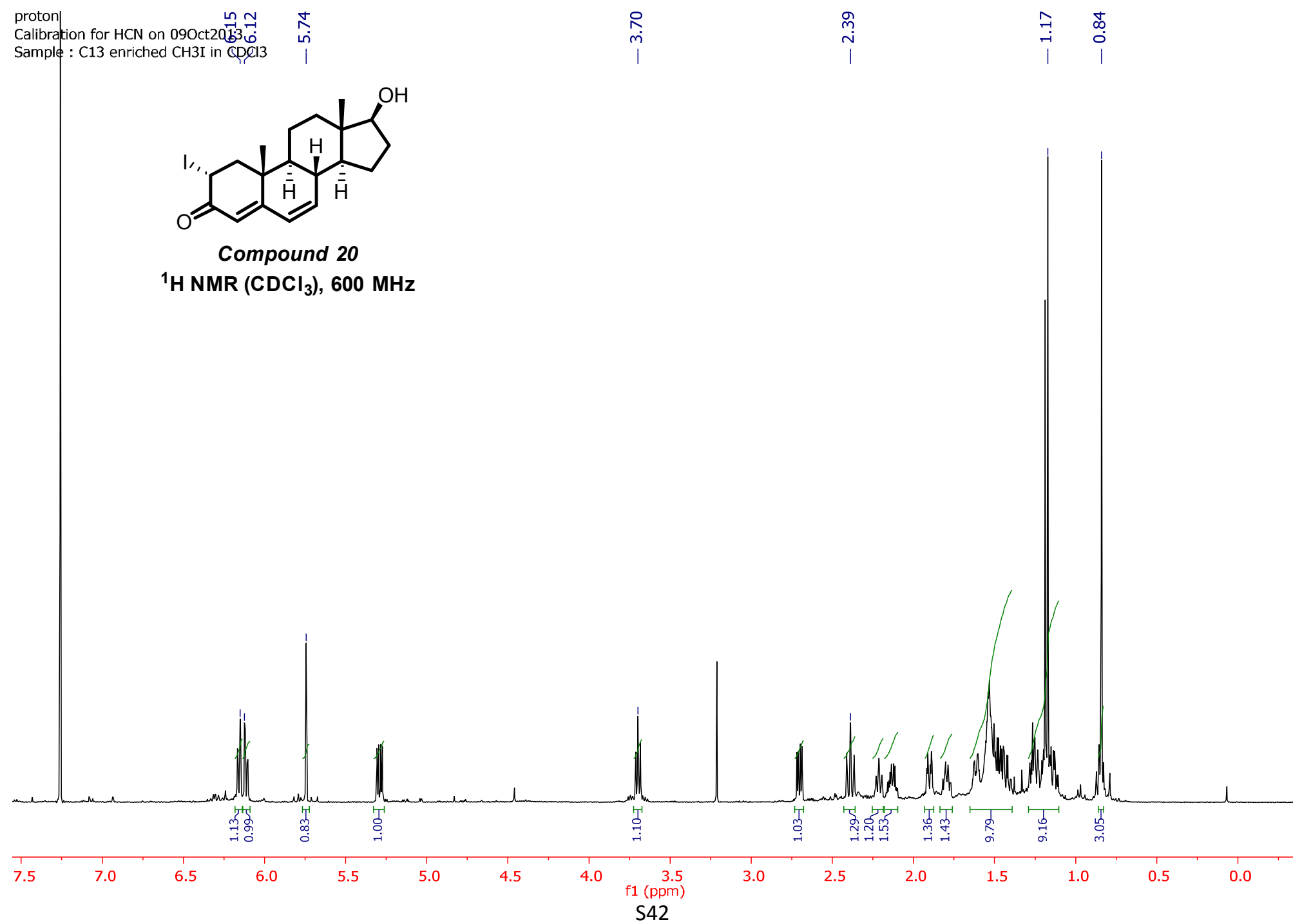




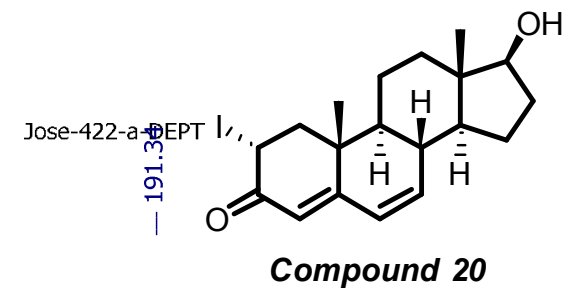

$\left.\left.{ }^{13} \mathrm{C} \mathrm{NMR} \mathrm{(CDCl}\right)_{3}\right), 75 \mathrm{MHz}$

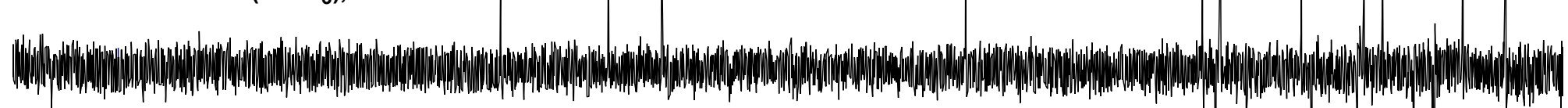

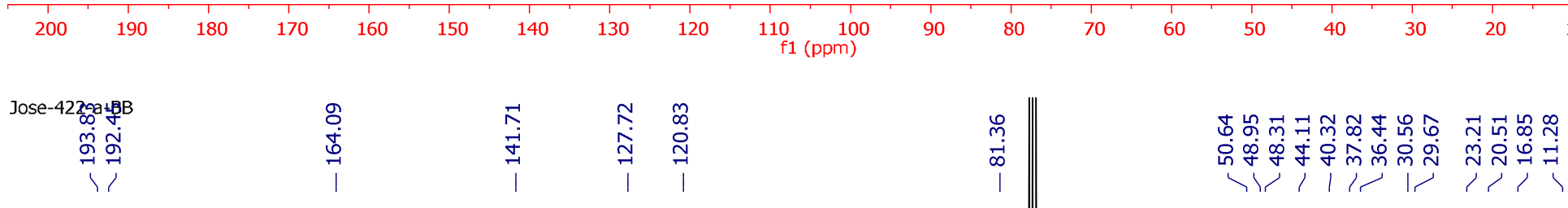

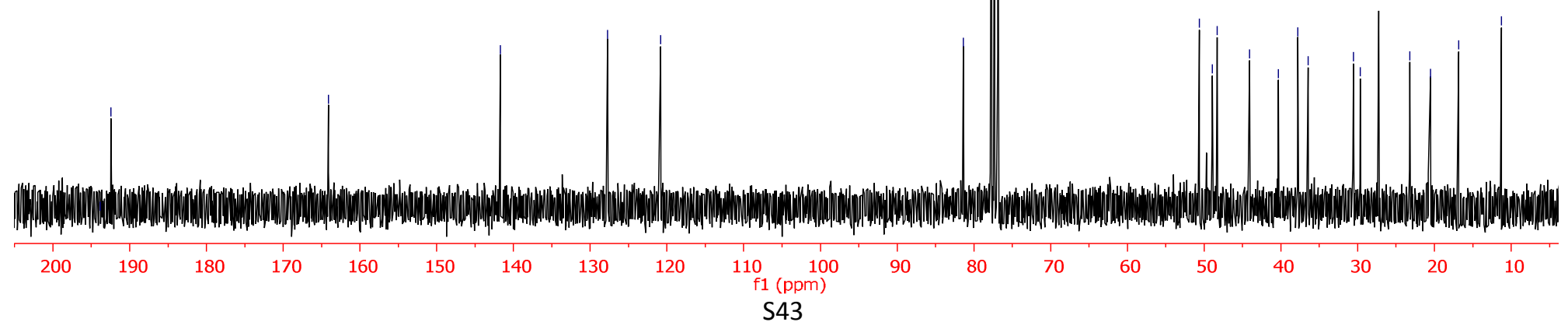




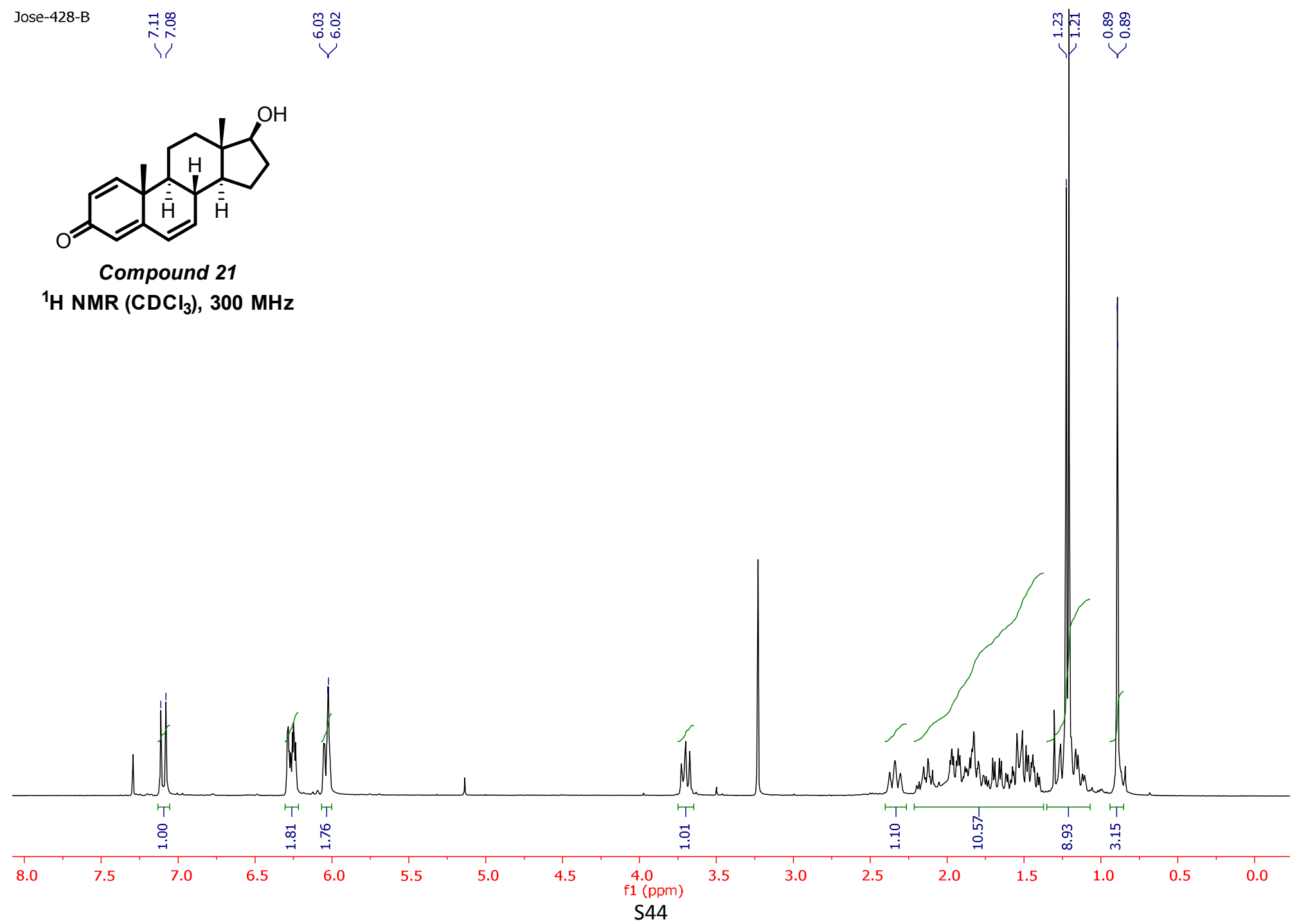




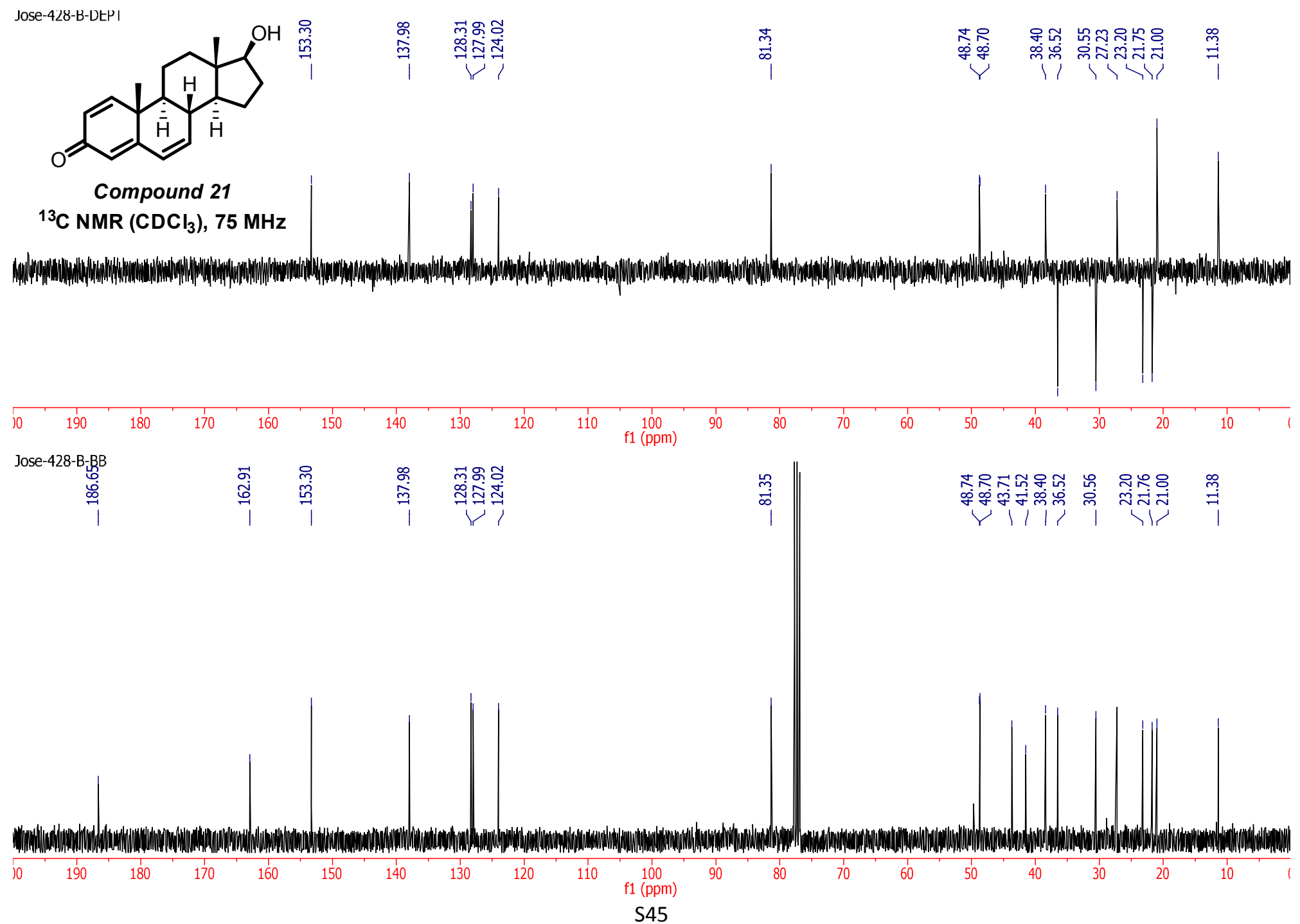

